KEK-TH-748

KUNS-1708

hep-th/0102168

Feb 2001

\title{
Supermatrix Models
}

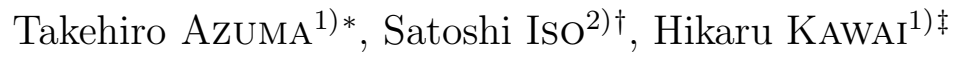 \\ and Yuhi OHWASHI ${ }^{1), 3}$ ) \\ 1) Department of Physics, Kyoto University, Kyoto 606-8502, Japan \\ 2) High Energy Accelerator Research Organization (KEK), \\ 1-1Oho, Tsukuba, Ibaraki 305-0801, Japan \\ 3) Department of Accelerator Science, \\ The Graduate University for Advanced Studies, \\ 1-1Oho, Tsukuba, Ibaraki 305-0801, Japan
}

\begin{abstract}
We investigate several matrix models based on super Lie algebras, osp $(1 \mid 32, R), u(1 \mid 16,16)$ and $g l(1 \mid 32, R)$. They are natural generalizations of IIB matrix model and were first proposed by Smolin [15]. In particular, we study the supersymmetry structures of these models and discuss possible reductions to IIB matrix model. We also point out that diffeomorphism invariance is hidden in gauge theories on noncommutative space which are derived from matrix models. This symmetry is independent of the global $S O(9,1)$ invariance in IIB matrix model and we report our trial to extend the global Lorentz invariance to local symmetry by introducing $u(1 \mid 16,16)$ or $g l(1 \mid 32, R)$ super Lie algebras.
\end{abstract}

\footnotetext{
* e-mail address : azuma@gauge.scphys.kyoto-u.ac.jp

$\dagger$ e-mail address : satoshi.iso@kek.jp

$\ddagger$ e-mail address : hkawai@gauge.scphys.kyoto-u.ac.jp

$\S$ e-mail address : yuhi@gauge.scphys.kyoto-u.ac.jp
} 


\section{Introduction}

A large $N$ reduced model has been proposed as a nonperturbative formulation of type IIB superstring theory [1] [2]. It is defined by the following action:

$$
S=-\frac{1}{g^{2}} \operatorname{Tr}\left(\frac{1}{4}\left[A_{\mu}, A_{\nu}\right]\left[A^{\mu}, A^{\nu}\right]+\frac{1}{2} \bar{\psi} \Gamma^{\mu}\left[A_{\mu}, \psi\right]\right) .
$$

It is a large $N$ reduced model [3] of 10 -dimensional $\mathcal{N}=1$ super Yang-Mills theory. Here $\psi$ is a 10-dimensional Majorana-Weyl spinor field, and $A_{\mu}$ and $\psi$ are $N \times N$ Hermitian matrices. This model is called IIB or IKKT matrix model. It is formulated in a manifestly covariant way which enables us to study the nonperturbative issues of superstring theory. In fact we can in principle predict the dimensionality of spacetime, the gauge group and the matter contents by solving this model. Such possibilities have been discussed in [4] [5]. We refer a review for more detailed expositions and references 6].

Although we have not yet obtained the complete interpretation of the model as the theory of gravity, the following arguments on supersymmetry lead us to interpret distributed eigenvalues as the extent of space-time. In addition to the original supersymmetry of the $\mathcal{N}=1$ super Yang-Mills

$$
\begin{aligned}
\delta^{(1)} \psi & =\frac{i}{2}\left[A_{\mu}, A_{\nu}\right] \Gamma^{\mu \nu} \epsilon, \\
\delta^{(1)} A_{\mu} & =i \bar{\epsilon} \Gamma^{\mu} \psi,
\end{aligned}
$$

the reduced model action (1.1) is invariant under the following shift of the fermionic variable

$$
\begin{aligned}
\delta^{(2)} \psi & =\xi \\
\delta^{(2)} A_{\mu} & =0 .
\end{aligned}
$$

Since the original 10-dimensional space-time is reduced to a single point, only the repetitions of the first transformations can no longer generate space-time translation. (It vanishes up to $S U(N)$ transformation.) However, if we take a linear combination of $\delta^{(1)}$ and $\delta^{(2)}$ as

$$
\begin{aligned}
& \tilde{\delta}^{(1)}=\delta^{(1)}+\delta^{(2)}, \\
& \tilde{\delta}^{(2)}=i\left(\delta^{(1)}-\delta^{(2)}\right),
\end{aligned}
$$

we obtain an enhanced $\mathcal{N}=2$ supersymmetry algebra

$$
\begin{aligned}
\left(\tilde{\delta}_{\epsilon}^{(i)} \tilde{\delta}_{\xi}^{(j)}-\tilde{\delta}_{\xi}^{(j)} \tilde{\delta}_{\epsilon}^{(i)}\right) \psi & =0 \\
\left(\tilde{\delta}_{\epsilon}^{(i)} \tilde{\delta}_{\xi}^{(j)}-\tilde{\delta}_{\xi}^{(j)} \tilde{\delta}_{\epsilon}^{(i)}\right) A_{\mu} & =-2 i \bar{\epsilon} \Gamma^{\mu} \xi \delta_{i j} .
\end{aligned}
$$


The r.h.s. is a shift of the bosonic variables

$$
\delta A^{\mu}=c^{\mu}
$$

where $c_{\mu}$ is proportional to unit matrix. The reduced model action (1.1) is, of course, invariant under this shift. Hence, if we interpret the bosonic variables as space-time coordinates, the above $\mathcal{N}=2$ supersymmetry generates translation in the new space-time.

Another reason to consider the bosonic variables $A^{\mu}$ as the space-time comes from the relation between matrix models and field theory on noncommutative geometry. As have been investigated in papers [7], matrix models can be rewritten as gauge theories on noncommutative space by expanding the bosonic variables $A^{\mu}$ around the noncommutative background $\hat{x}_{\mu}$ satisfying

$$
\left[\hat{x}^{\mu}, \hat{x}^{\nu}\right]=-i \theta^{\mu \nu}
$$

where $\theta^{\mu \nu}$ are $c$-numbers. We assume the rank of $\theta^{\mu \nu}$ to be $\tilde{d}$ and define its inverse $\beta_{\mu \nu}$ in $\tilde{d}$-dimensional subspace. $\hat{x}^{\mu}$ satisfy the canonical commutation relations and they span the $\tilde{d}$-dimensional phase space. The semiclassical correspondence shows that the volume of the phase space (measured in the coordinate space of $x^{\mu}$ ) is $V=N(2 \pi)^{\tilde{d} / 2} \sqrt{\operatorname{det} \theta}$. Distribution of eigenvalues of $\hat{x}$ is therefore interpreted as space-time. In noncommutative space, space-time translation can be generated by unitary transformation of $A_{\mu}$. Furthermore, the dynamical generation of space-time implies that the fluctuation of space-time is also dynamical and graviton will be hidden in IIB matrix model or equivalently in noncommutative gauge theory. Investigations of noncommutative gauge theories have indeed clarified that they can contain much larger degrees of freedom than those in the ordinary commutative field theories. For example, noncommutative plane waves are interpreted as bi-local rather than local [8]. After they are expanded in terms of local operators it is expected that higher spin fields will appear, even if we start from Yang-Mills theory. From this point of view, we expect that noncommutative Yang-Mills can contain graviton. A possible interpretation of diffeomorphism invariance in noncommutative Yang-Mills is given in section 3 in this paper.

In these ways, matrix models can describe both space-time and matter, i.e. fluctuation around a classical background, in the same footing. Such a unification seems only possible in the case of gauge theory where bosonic fields have the same indices as the space-time. But the unification of space-time and matter is so far restricted to a flat space-time and a natural question is how we can describe a curved space-time in matrix models. (A simple example for the fuzzy sphere is discussed from the matrix model point of view in [9].) In 
the flat $\tilde{d}$-dimensional space discussed above, we can identify some of the $S O(9,1)$ indices with the indices of $S O(\tilde{d})$ isometries of the background. But this cannot be expected for more general curved backgrounds whose isometries cannot be embedded in $S O(9,1)$. If IIB matrix model is a background independent model, general coordinate transformations will be hidden and the $S O(9,1)$ symmetries should be rather considered as a gauge fixed local Lorentz symmetry instead of isometry of 10-dimensional flat space-time. (A possible interpretation of diffeomorphism in IIB matrix model is discussed in [10].) One way to reveal such a structure will be to find an extended model with larger symmetries that reproduces IIB matrix model after gauge fixing and integrating irrelevant fields. In order that this model can describe a curved space-time, a spin connection term containing a $\gamma$-matrix of rank 3 must be included or generated in the fermionic action.

Following the above discussions we search for models with higher rank tensor fields coupled to fermions through $\gamma$-matrices. Another guiding principle to construct a model is a sufficient number of supersymmetries. In order to reproduce IIB matrix model we need at least 10 -dimensional $\mathcal{N}=2$ supersymmetry. These requirements can be satisfied by considering matrix models based on super Lie algebra $\operatorname{ssp}(1 \mid 32, R)$. This superalgebra was mentioned first on 11-dimensional supergravity [11] and investigated systematically in [12]. It has attracted a new attention as the unified superalgebra for M-theory in [13] [14]. Construction of matrix models based on this superalgebra was proposed by Smolin [15].

In this paper, we investigate such models, especially from the point of supersymmetries. In section 2, we consider a model based on $\operatorname{osp}(1 \mid 32, R)$ super Lie algebra. Bosonic fields in this model can be expanded in terms of 11-dimensional $\gamma$-matrices of rank 1, 2 and 5 . They are real fields. Fermionic fields are composed of 11-dimensional Majorana fermion. Hence, reduced to $\mathrm{d}=10$, this model becomes vector-like and we have to integrate out a right(or left)-handed sector in order to reproduce IIB matrix model. The symmetry of this model is a direct product of $O S p(1 \mid 32, R)$ and $U(N)$. The $O S p(1 \mid 32, R)$ group is a generalization of $S O(9,1)$ in IIB matrix model. The model is also invariant under constant shifts of fields and we show that the $\operatorname{OSp}(1 \mid 32, R)$ symmetry and the constant shift of fields are combined to form space-time algebras including space-time supersymmetry. We discuss a possibility to obtain IIB matrix model by integrating some of the fields.

In section 3, we study how diffeomorphism invariance is hidden in IIB matrix model. We first give a brief summary of the relation between matrix models and gauge theories on noncommutative space. We then show that the unitary gauge transformation is much larger 
in noncommutative space than that in ordinary commutative space. Even local coordinate transformations are generated by the unitary transformations. It is also pointed out that this diffeomorphism invariance is independent of the global $S O(9,1)$ invariance and it is difficult to extend the global $S O(9,1)$ to local symmetry of the model.

To search for extended models with local Lorentz invariance, we then construct matrix models with local $S O(9,1)$ symmetry in section 4 . In particular, we investigate matrix models based on $u(1 \mid 16,16)$ or $g l(1 \mid 32, R)$ super Lie algebra. These models are invariant under coupled symmetries of $U(1 \mid 16,16)$ (or $G L(1 \mid 32, R)$ ) and $U(N)$. Since $U(1 \mid 16,16)$ (or $G L(1 \mid 32, R))$ is an extension of $S O(9,1)$, this model has local (i.e. $U(N)$-dependent) Lorentz invariance. At the cost of this enhanced gauge symmetries, these models break invariance under constant shifts of fields and we need another interpretation of space-time translation. We make use of the Wigner-Inönü contraction and identify generators of $S O(10,1)$ (which is a subgroup of $U(1 \mid 16,16)$ and $G L(1 \mid 32, R))$ with generators of $S O(9,1)$ rotations and translations in 10-dimensional space-time. In this way, we can obtain 10-dimensional spacetime picture. We also determine how to scale the fields to obtain the correct 10-dimensional theory.

The final section is devoted to conclusions and discussions.

\section{$2 \operatorname{osp}(1 \mid 32, R)$ Cubic Matrix Model}

Smolin proposed a matrix model based on the super Lie algebra $\operatorname{csp}(1 \mid 32, R)$ [15] as an M-theory matrix model. The action is constructed from $\operatorname{osp}(1 \mid 32, R)$ matrix $M$ whose components are also $N \times N$ matrices. The bosonic part of this model can be expanded in terms of 11-dimensional $\gamma$-matrices with rank 1, 2 and 5 . Therefore it is a natural extension of ordinary matrix models containing only vector field with rank 1 . Furthermore it has a simple cubic form in terms of matrix $M$ and is reminiscent of Witten's string field theory. Before going into detailed analysis of the model, we first give the definitions of $\operatorname{osp}(1 \mid 32, R)$ super Lie algebra.

\subsection{Definition of $\operatorname{osp}(1 \mid 32, R)$ supermatrix}

$\operatorname{osp}(1 \mid 32, R)$ super matrix is a $33 \times 33$ real supermatrix satisfying the following conditions:

$$
\begin{aligned}
& { }^{T} M G+G M=0 \text { for } G=\left(\begin{array}{cc}
\Gamma^{0} & 0 \\
0 & i
\end{array}\right), \\
& M^{*}=M
\end{aligned}
$$


$\Gamma^{0}$ is a $32 \times 32$ 11-dimensional $\gamma$-matrix in Majorana basis which is real and satisfies $\left(\Gamma^{0}\right)^{2}=$ -1 . For conventions of a super matrix, see Appendix A. An element of $O S p(1 \mid 32, R)$ group is written as $U=\exp (M)$ and satisfies

$$
{ }^{T} U G U=G .
$$

The above conditions (2.1) restrict the matrix $M$ to be

$$
M=\left(\begin{array}{cc}
m & \psi \\
i \bar{\psi} & 0
\end{array}\right),
$$

where $\psi$ is a Majorana spinor with 32 components and $\bar{\psi}={ }^{T} \psi \Gamma^{0} . m$ is a real $32 \times 32$ bosonic matrix satisfying

$$
{ }^{T} m \Gamma^{0}+\Gamma^{0} m=0 .
$$

The bosonic part $m$ is an element of $\operatorname{sp}(32, R)$ algebra. It can be expanded in terms of 11-dimensional $\gamma$-matrices as

$$
m=u_{A_{1}} \Gamma^{A_{1}}+\frac{1}{2 !} u_{A_{1} A_{2}} \Gamma^{A_{1} A_{2}}+\frac{1}{5 !} u_{A_{1} \cdots A_{5}} \Gamma^{A_{1} \cdots A_{5}},
$$

and contains $528=11+55+462$ degrees of freedom. $A_{i}$ are denoted as 11-dimensional indices and run from 0 to 10 . We are working in Majorana basis, where all $\gamma$-matrices are real. Therefore the real condition means that all the coefficients $u_{A_{1}}, u_{A_{1} A_{2}}$ and $u_{A_{1} \cdots A_{5}}$ are real.

\subsection{Action and symmetries}

In considering the action of a large $N$ reduced model, we regard each of the coefficients $u_{A_{1}}, u_{A_{1} A_{2}}$ and $u_{A_{1} \cdots A_{5}}$ and each component of $\psi$ as an $N \times N$ hermitian matrix. $M_{P}{ }^{Q}$, each component of the supermatrix $M$, is thus an $N \times N$ hermitian matrix. We further introduce $N^{2} \operatorname{osp}(1 \mid 32, R)$ supermatrices $M^{a}$ as the coefficients of $M$ expanded in terms of the Gell-Mann matrices $t^{a}$ :

$$
M=\sum_{a=1}^{N^{2}} t^{a} M^{a} .
$$

The action proposed by Smolin is

$$
\begin{aligned}
I & =\frac{i}{g^{2}} \operatorname{Tr}_{N \times N} \sum_{Q, R=1}^{33}\left(\left(\sum_{p=1}^{32} M_{p}{ }^{Q}\left[M_{Q}^{R}, M_{R}^{p}\right]\right)-M_{33}{ }^{Q}\left[M_{Q}^{R}, M_{R}^{33}\right]\right) \\
& =\frac{i}{g^{2}} \sum_{a, b, c=1}^{N^{2}} \operatorname{Str}_{33 \times 33}\left(M^{a} M^{b} M^{c}\right) \operatorname{Tr}_{N \times N}\left(t^{a}\left[t^{b}, t^{c}\right]\right),
\end{aligned}
$$


where $p=1, \cdots, 32, P, Q, R=1,2, \cdots 33$ and $a, b, c$ are indices for $U(N)$. To avoid confusions, we note here that we use $\operatorname{Tr}$ as a trace of $N \times N$ matrices while $\operatorname{tr}$ (or Str) as a trace of $32 \times 32(33 \times 33$ super $)$ matrices. This action can be rewritten as

$$
\begin{aligned}
I & =-\frac{f_{a b c}}{2 g^{2}}\left(\operatorname{tr}_{32 \times 32}\left(m^{a} m^{b} m^{c}\right)-3 i \bar{\psi}^{a} m^{b} \psi^{c}\right) \\
& \left.=\frac{i}{g^{2}} \operatorname{Tr}_{N \times N}\left(m_{p}{ }^{q}\left[m_{q}{ }^{r}, m_{r}{ }^{p}\right]\right)-3 i \bar{\psi}^{p}\left[m_{p}{ }^{q}, \psi_{q}\right]\right) .
\end{aligned}
$$

$f_{a b c}$ are structure constants defined by $\left[t_{a}, t_{b}\right]=i f_{a b c} t_{c}$. The fermionic term has the same form as that of IIB matrix model but the bosonic part is cubic and different. This difference is related to the difference in supersymmetry. That is, in the IIB case the supersymmetry transformation (1.2) for $\psi$ is proportional to a commutator of the bosonic field $\left[A_{\mu}, A_{\nu}\right]$ while here all (homogeneous) transformations are linear in fields as we will see soon. Another big difference is that this model contains 32 component Majorana fermion compared to 16 in IIB matrix model. Due to this doubling, we need to integrate out half of fermions in order to show the equivalence to IIB matrix model.

In spite of these differences, this model possesses several similarities. First it has no free parameter since the coupling constant is always absorbed by a field redefinition of matrix $M$. Hence $g$ gives the only dimensionful parameter in the model. Symmetries of the model also have similar structures to IIB matrix model. If we write the matrix $M$ as a tensor product of $\operatorname{osp}(1 \mid 32, R)$ and $N \times N$ matrix, the action is invariant under

$$
M \rightarrow\left(U \otimes 1_{N \times N}\right) M\left(U \otimes 1_{N \times N}\right)^{-1}
$$

where $U$ is an element of $O S p(1 \mid 32, R)$ group. For an infinitesimal transformation,

$$
\delta M=[H, M]=\left[\left(\begin{array}{cc}
h & \chi \\
i \bar{\chi} & 0
\end{array}\right),\left(\begin{array}{cc}
m & \psi \\
i \bar{\psi} & 0
\end{array}\right)\right]=\delta_{h} M+\delta_{\chi}^{(1)} M
$$

The bosonic part is identified with $s p(32, R)$ rotations:

$$
\delta_{h} M=\left(\begin{array}{cc}
{[h, m]} & h \psi \\
i \bar{\psi} h & 0
\end{array}\right) .
$$

The fermionic part, supersymmetry transformation, is given by

$$
\delta_{\chi}^{(1)} M=\left(\begin{array}{cc}
i(\chi \bar{\psi}-\psi \bar{\chi}) & -m \chi \\
i \bar{\chi} m & 0
\end{array}\right) .
$$

The bosonic part is a natural extension of $S O(9,1)$ rotation in IIB matrix model and indeed includes $S O(10,1)$ symmetry. Besides this $S O(10,1)$ symmetry generated by $\Gamma^{A_{1} A_{2}}$, 
there are bosonic symmetries generated by $\gamma$-matrices with rank 1 and 5 . These transformations mix bosonic fields with a different number of 11-dimensional indices. The fermionic part is a generalization of homogeneous supersymmetry (1.2) in IIB matrix model. As already mentioned, this homogeneous supersymmetry is linear in all fields and the action is invariant under this supersymmetry among terms with the same number of fields. This is different from IIB matrix model where the action is balanced between $\operatorname{Tr}_{N \times N}\left[A_{\mu}, A_{\nu}\right]^{2}$ and $\operatorname{Tr}_{N \times N} \bar{\psi} \gamma^{\mu}\left[A_{\mu}, \psi\right]$ under supersymmetry since the transformation for the fermion contains two bosonic fields. We expect that, by integrating some of the fields, the supersymmetry structure of IIB matrix model may be reproduced from this osp $(1 \mid 32, R)$ model.

The action is also invariant under $U(N)$ symmetry

$$
M \rightarrow\left(1_{33 \times 33} \otimes U\right) M\left(1_{33 \times 33} \otimes U\right)^{-1},
$$

where $U$ is an element of $U(N)$ group. All the $\operatorname{osp}(1 \mid 32, R)$ fields must be transformed simultaneously. The symmetry of our model is therefore a direct product of these two Lie groups $O S p(1 \mid 32, R) \times U(N)$.

Another symmetry of the model is a trivial shift of the supermatrix $M$ :

$$
M_{P}^{Q} \rightarrow M_{P}^{Q}+c_{P}{ }^{Q} 1_{N \times N}
$$

This shift contains both bosonic and fermionic inhomogeneous transformations. Some of the bosonic shifts are identified with space-time translations while the fermionic shifts form space-time supersymmetry together with the fermionic part of the homogeneous osp $(1 \mid 32, R)$ transformations. We write down the fermionic part explicitly for later convenience:

$$
\delta_{\epsilon}^{(2)} m=0, \quad \delta_{\epsilon}^{(2)} \psi=\epsilon .
$$

Summarizing the three kinds of symmetries, the bosonic invariance of the model contains $S p(32, R)$ rotation with 528 generators, constant shifts for each $528 \operatorname{sp}(32, R)$ fields and $U(N)$ gauge symmetry. The fermionic invariance, i.e. supersymmetry, is generated by homogeneous supersymmetry transformations (2.12) with real 32 components and inhomogeneous transformations (2.15) with the same number of components.

We then study the algebraic structures of these symmetries . The commutation relations among the homogeneous supersymmetries (2.12) are, of course, written by $S p(32, R)$ rotations:

$$
\begin{aligned}
& {\left[\delta_{\chi}^{(1)}, \delta_{\epsilon}^{(1)}\right] m=i[(\chi \bar{\epsilon}-\epsilon \bar{\chi}), m]} \\
& {\left[\delta_{\chi}^{(1)}, \delta_{\epsilon}^{(1)}\right] \psi=i(\chi \bar{\epsilon}-\epsilon \bar{\chi}) \psi}
\end{aligned}
$$


$h=i(\chi \bar{\epsilon}-\epsilon \bar{\chi})$ is an element of $\operatorname{sp}(32, R)$ and can be expanded as

$$
h=h_{A} \Gamma^{A}+\frac{1}{2 !} h_{A_{1} A_{2}} \Gamma^{A_{1} A_{2}}+\frac{1}{5 !} h_{A_{1} \cdots A_{5}} \Gamma^{A_{1} \cdots A_{5}},
$$

where

$$
h_{A}=\frac{1}{32} \operatorname{tr}\left(h \Gamma_{A}\right), \quad h_{A_{1} A_{2}}=-\frac{1}{32} \operatorname{tr}\left(h \Gamma_{A_{1} A_{2}}\right), \quad h_{A_{1} \cdots A_{5}}=\frac{1}{32} \operatorname{tr}\left(h \Gamma_{A_{1} \cdots A_{5}}\right) .
$$

It has the same algebraic structure as the 11-dimensional space-time supersymmetry with central charges of rank 2 and 5. But this algebra itself can no longer be interpreted as spacetime supersymmetry since transformations generated by $\Gamma^{A}$ are not the translation of spacetime. The situation is the same as in IIB matrix model. If we interpret eigenvalues of some bosonic variables as our space-time coordinates, space-time translation should be identified with the constant shift of bosonic fields. A difference is that, in IIB matrix model, this type of commutation relation vanishes up to a field dependent $U(N)$ gauge transformation while here we have $\operatorname{sp}(32, R)$ rotations.

Commutation relations between the homogeneous and inhomogeneous supersymmetry transformations are, on the other hand, given by

$$
\left[\delta_{\chi}^{(1)}, \delta_{\epsilon}^{(2)}\right] m=-i(\chi \bar{\epsilon}-\epsilon \bar{\chi}), \quad\left[\delta_{\chi}^{(1)}, \delta_{\epsilon}^{(2)}\right] \psi=0
$$

and generate a constant shift of bosonic fields. Commutators between inhomogeneous transformations trivially vanish.

By taking linear combinations as

$$
\begin{aligned}
& \tilde{\delta}^{(1)}=\delta^{(1)}+\delta^{(2)}, \\
& \tilde{\delta}^{(2)}=i\left(\delta^{(1)}-\delta^{(2)}\right),
\end{aligned}
$$

we obtain an enhanced 'space-time' supersymmetry algebra

$$
\begin{aligned}
{\left[\tilde{\delta}_{\chi}^{(i)}, \tilde{\delta}_{\epsilon}^{(j)}\right] m } & =-2 i(\chi \bar{\epsilon}-\epsilon \bar{\chi}) \delta_{i j} \\
{\left[\tilde{\delta}_{\chi}^{(i)}, \tilde{\delta}_{\epsilon}^{(j)}\right] \psi } & =0
\end{aligned}
$$

up to $s p(32, R)$ rotations. As far as the supersymmetry algebra is concerned, $s p(32, R)$ transformations are more appropriately interpreted as a kind of gauge symmetries. 


\subsection{Reduction to $d=10$}

So far we have studied the model from 11-dimensional point of view. In this subsection, we investigate it from 10-dimensional point of view by specializing the 10th direction. For this purpose, we first introduce the following new variables

$$
\begin{aligned}
& W=u_{\sharp}, \quad A_{\mu}^{( \pm)}=u_{\mu} \pm u_{\mu \sharp}, \quad C_{\mu_{1} \mu_{2}}=u_{\mu_{1} \mu_{2}}, \\
& H_{\mu_{1} \cdots \mu_{4}}=u_{\mu_{1} \cdots \mu_{4} \sharp}, \quad I_{\mu_{1} \cdots \mu_{5}}^{( \pm)}=\frac{1}{2}\left(u_{\mu_{1} \cdots \mu_{5}} \pm \tilde{u}_{\mu_{1} \cdots \mu_{5}}\right) .
\end{aligned}
$$

Here we use the indices $\mu_{1}, \mu_{2}, \cdots$ running from 0 to 9 . $\sharp$ denotes the 10 th direction. The quantity $\tilde{u}_{\mu_{1} \cdots \mu_{5}}$ denotes the dual of $u_{\mu_{1} \cdots \mu_{5}}$ :

$$
\tilde{u}_{\mu_{1} \cdots \mu_{5}}=\frac{-1}{5 !} u_{\mu_{6} \cdots \mu_{10}} \epsilon^{\mu_{1} \cdots \mu_{10 \sharp}} .
$$

$I_{\mu_{1} \cdots \mu_{5}}^{(+)}$and $I_{\mu_{1} \cdots \mu_{5}}^{(-)}$are self-dual and anti-self-dual respectively:

$$
I_{\mu_{1} \cdots \mu_{5}}^{( \pm)}= \pm \tilde{I}_{\mu_{1} \cdots \mu_{5}}^{( \pm)}
$$

Looking at these fields, we have two set of fields $A_{\mu}^{( \pm)}$that can be identified with $A_{\mu}$ field in IIB matrix model. This is in accord with the doubling of fermions. These doublings cannot be avoided since we start from a 11-dimensional model. It is now convenient to define an even rank bosonic field and two odd rank fields $m_{o}{ }^{( \pm)}$by

$$
\begin{aligned}
m_{e} & =W \Gamma^{\sharp}+\frac{1}{2} C_{\mu \nu} \Gamma^{\mu \nu}+\frac{1}{4 !} H_{\mu_{1} \cdots \mu_{4}} \Gamma^{\mu_{1} \cdots \mu_{4}}, \\
m_{o}^{(+)} & =\left(\frac{1}{2} A_{\mu}^{(+)} \Gamma^{\mu}+\frac{1}{5 !} I_{\mu_{1} \cdots \mu_{5}}^{(+)} \Gamma^{\mu_{1} \cdots \mu_{5}}\right)\left(1+\Gamma^{\sharp}\right), \\
m_{o}^{(-)} & =\left(\frac{1}{2} A_{\mu}^{(-)} \Gamma^{\mu}+\frac{1}{5 !} I_{\mu_{1} \cdots \mu_{5}}^{(-)} \Gamma^{\mu_{1} \cdots \mu_{5}}\right)\left(1-\Gamma^{\sharp}\right) .
\end{aligned}
$$

Fermions are also decomposed into left and right handed chiralities

$$
\psi_{L}=\frac{1+\Gamma^{\sharp}}{2} \psi, \quad \psi_{R}=\frac{1-\Gamma^{\sharp}}{2} \psi .
$$

Here we note the following useful identities:

$$
m_{o}^{(+)} \psi_{R}=m_{o}^{(-)} \psi_{L}=0, \quad \bar{\psi}_{R} m_{o}^{(+)}=\bar{\psi}_{L} m_{o}^{(-)}=0
$$

If we denote sets of the fields $m_{e}, m_{o}^{( \pm)}$by $\mathcal{M}_{e}, \mathcal{M}_{o}^{( \pm)}$, we also have the relations

$$
\begin{aligned}
& \chi_{L} \bar{\epsilon}_{L} \in \mathcal{M}_{o}^{(-)}, \chi_{R} \bar{\epsilon}_{R} \in \mathcal{M}_{o}^{(+)}, \chi_{L} \bar{\epsilon}_{R} \in \mathcal{M}_{e}, \chi_{R} \bar{\epsilon}_{L} \in \mathcal{M}_{e} \\
& {\left[\mathcal{M}_{e}, \mathcal{M}_{e}\right] \in \mathcal{M}_{e},\left[\mathcal{M}_{e}, \mathcal{M}_{o}^{( \pm)}\right] \in \mathcal{M}_{o}^{( \pm)},\left[\mathcal{M}_{o}^{(+)}, \mathcal{M}_{o}^{(-)}\right] \in \mathcal{M}_{e}} \\
& \mathcal{M}_{o}^{(+)} \mathcal{M}_{o}^{(+)}=0, \mathcal{M}_{o}^{(-)} \mathcal{M}_{o}^{(-)}=0 .
\end{aligned}
$$


Then the action $I=I_{b}+I_{f}$ becomes

$$
\begin{aligned}
I_{b} & =\frac{-f_{a b c}}{2 g^{2}} \operatorname{tr}_{32 \times 32}\left[m_{e}^{a} m_{e}^{b} m_{e}^{c}+3 m_{e}^{a} m_{o}^{(+) b} m_{o}^{(-) c}+3 m_{e}^{a} m_{o}^{(-) b} m_{o}^{(+) c}\right] \\
I_{f} & =\frac{3 i f_{a b c}}{2 g^{2}}\left[2 \bar{\psi}_{L}^{a} m_{e}^{b} \psi_{R}^{c}+\bar{\psi}_{L}^{a} m_{o}^{(+) b} \psi_{L}^{c}+\bar{\psi}_{R}^{a} m_{o}^{(-) b} \psi_{R}^{c}\right] .
\end{aligned}
$$

The structure is very simple. There are two sectors $\left(\psi_{L}\right.$ and $\left.m_{o}^{(+)}\right)$and $\left(\psi_{R}\right.$ and $\left.m_{o}^{(-)}\right)$which are coupled through $m_{e}$ fields. We can then expect to obtain IIB like model if we succeed in integrating one sector. The situation is unfortunately more complicated as we will see in the following discussions of supersymmetries. Here we write down the action in terms of 10-dimensional components for later purpose:

$$
\begin{aligned}
& I_{b}=\frac{i}{g^{2}} \operatorname{Tr}_{N \times N}\left(-96\left[A_{\mu_{1}}^{(+)}, A_{\mu_{2}}^{(-)}\right] C^{\mu_{1} \mu_{2}}-96 W\left[A^{(+) \mu}, A_{\mu}^{(-)}\right]+\frac{4}{5} W\left[I_{\mu_{1} \cdots \mu_{5}}^{(+)}, I^{(-) \mu_{1} \cdots \mu_{5}}\right]\right. \\
& -4 H_{\mu_{1} \cdots \mu_{4}}\left(\left[A_{\mu_{5}}^{(+)}, I^{(-) \mu_{1} \cdots \mu_{5}}\right]-\left[A_{\mu_{5}}^{(-)}, I^{(+) \mu_{1} \cdots \mu_{5}}\right]\right)-8 C_{\mu_{1} \mu_{2}}\left[I^{(+) \mu_{1}}{ }_{\mu_{3} \cdots \mu_{6}}, I^{(-) \mu_{2} \cdots \mu_{6}}\right] \\
& +\frac{8}{3} H_{\mu_{1} \mu_{2}}^{\nu \lambda}\left(\left[I^{(+)}{ }_{\nu \lambda \mu_{3} \mu_{4} \mu_{5}}, I^{(-) \mu_{1} \cdots \mu_{5}}\right]-\left[I_{\nu \lambda \mu_{3} \mu_{4} \mu_{5}}^{(-)}, I^{(+) \mu_{1} \cdots \mu_{5}}\right]\right) \\
& +32\left[C_{\mu_{2}}^{\mu_{1}}, C_{\mu_{1} \mu_{3}}\right] C^{\mu_{2} \mu_{3}}-16 C_{\mu_{1} \mu_{2}}\left[H_{\mu_{3} \mu_{4} \mu_{5}}^{\mu_{1}}, H^{\mu_{2} \cdots \mu_{5}}\right] \\
& \left.+\frac{1}{27} H_{\mu_{1} \cdots \mu_{4}}\left[H_{\mu_{5} \cdots \mu_{7}}^{\nu}, H_{\nu \mu_{8} \mu_{9} \mu_{10}}\right] \epsilon^{\mu_{1} \cdots \mu_{10} \sharp}\right) \text {, } \\
& I_{f}=\frac{i}{g^{2}} \operatorname{Tr}_{N \times N}\left(-3 i\left(-\bar{\psi}_{L}\left[W, \psi_{R}\right]+\bar{\psi}_{R}\left[W, \psi_{L}\right]\right)\right. \\
& -3 i\left(\bar{\psi}_{L} \Gamma^{i}\left[A_{\mu}^{(+)}, \psi_{L}\right]+\bar{\psi}_{R} \Gamma^{\mu}\left[A_{\mu}^{(-)}, \psi_{R}\right]\right) \\
& -\frac{3 i}{2 !}\left(\bar{\psi}_{L} \Gamma^{\mu_{1} \mu_{2}}\left[C_{\mu_{1} \mu_{2}}, \psi_{R}\right]+\bar{\psi}_{R} \Gamma^{\mu_{1} \mu_{2}}\left[C_{\mu_{1} \mu_{2}}, \psi_{L}\right]\right) \\
& -\frac{3 i}{4 !}\left(-\bar{\psi}_{L} \Gamma^{\mu_{1} \mu_{2} \mu_{3} \mu_{4}}\left[H_{\mu_{1} \mu_{2} \mu_{3} \mu_{4}}, \psi_{R}\right]+\bar{\psi}_{R} \Gamma^{\mu_{1} \mu_{2} \mu_{3} \mu_{4}}\left[H_{\mu_{1} \mu_{2} \mu_{3} \mu_{4}}, \psi_{L}\right]\right) \\
& \left.-\frac{3 i}{5 !}\left(2 \bar{\psi}_{L} \Gamma^{\mu_{1} \mu_{2} \mu_{3} \mu_{4} \mu_{5}}\left[I_{\mu_{1} \mu_{2} \mu_{3} \mu_{4} \mu_{5}}^{(+)}, \psi_{L}\right]+2 \bar{\psi}_{R} \Gamma^{\mu_{1} \mu_{2} \mu_{3} \mu_{4} \mu_{5}}\left[I_{\mu_{1} \mu_{2} \mu_{3} \mu_{4} \mu_{5}}^{(-)}, \psi_{R}\right]\right)\right) .(2
\end{aligned}
$$

We then investigate the symmetry structures, especially the structures of supersymmetry, instead of explicitly integrating out some fields in order to see a possibility to induce IIB matrix model. We first perform chiral decomposition of both homogeneous and inhomogeneous supersymmetries. 64 supersymmetries are decomposed into 16 left(right)-handed homogeneous (inhomogeneous) supersymmetries:

$$
\delta_{\epsilon_{L . R}}^{(1)}, \quad \delta_{\epsilon_{L . R}}^{(2)}
$$

Under the homogeneous supersymmetries, the fields transform as

$$
\delta_{\chi}^{(1)} m_{e}=i\left(\chi_{L} \bar{\psi}_{R}-\psi_{L} \bar{\chi}_{R}+\chi_{R} \bar{\psi}_{L}-\psi_{R} \bar{\chi}_{L}\right)
$$




$$
\begin{array}{ll}
\delta_{\chi}^{(1)} m_{o}^{(+)}=i\left(\chi_{R} \bar{\psi}_{R}-\psi_{R} \bar{\chi}_{R}\right), & \delta_{\chi}^{(1)} \psi_{R}=-m_{e} \chi_{R}-m_{o}^{(+)} \chi_{L}, \\
\delta_{\chi}^{(1)} m_{o}^{(-)}=i\left(\chi_{L} \bar{\psi}_{L}-\psi_{L} \bar{\chi}_{L}\right), & \delta_{\chi}^{(1)} \psi_{L}=-m_{e} \chi_{L}-m_{o}^{(-)} \chi_{R} .
\end{array}
$$

Here a natural pairing is

$$
m_{o}^{(+)} \leftrightarrow \psi_{R}, \quad m_{o}^{(-)} \leftrightarrow \psi_{L},
$$

which is different from the pairing which appears in the action (2.30). The inhomogeneous supersymmetry transformations are trivial

$$
\begin{aligned}
\delta_{\epsilon}^{(2)} \psi_{L(R)} & =\epsilon_{L(R)}, \\
\delta_{\epsilon}^{(2)} m & =0 .
\end{aligned}
$$

The commutation relations between the homogeneous supersymmetry transformations are written in terms of even and odd fields as

$$
\begin{aligned}
{\left[\delta_{\chi}^{(1)}, \delta_{\epsilon}^{(1)}\right] m_{e}=} & i\left[\left(\chi_{L} \bar{\epsilon}_{R}-\epsilon_{L} \bar{\chi}_{R}+\chi_{R} \bar{\epsilon}_{L}-\epsilon_{R} \bar{\chi}_{L}\right), m_{e}\right] \\
& +i\left[\chi_{L} \bar{\epsilon}_{L}-\epsilon_{L} \bar{\chi}_{L}, m_{o}^{(+)}\right]+i\left[\chi_{R} \bar{\epsilon}_{R}-\epsilon_{R} \bar{\chi}_{R}, m_{o}^{(-)}\right], \\
{\left[\delta_{\chi}^{(1)}, \delta_{\epsilon}^{(1)}\right] m_{o}^{(+)}=} & i\left[\left(\chi_{R} \bar{\epsilon}_{R}-\epsilon_{R} \bar{\chi}_{R}\right), m_{e}\right]+i\left(\chi_{R} \bar{\epsilon}_{L}-\epsilon_{R} \bar{\chi}_{L}\right) m_{o}^{(+)}-i m_{o}^{(+)}\left(\chi_{L} \bar{\epsilon}_{R}-\epsilon_{L} \bar{\chi}_{R}\right), \\
{\left[\delta_{\chi}^{(1)}, \delta_{\epsilon}^{(1)}\right] m_{o}^{(-)}=} & i\left[\left(\chi_{L} \bar{\epsilon}_{L}-\epsilon_{L} \bar{\chi}_{L}\right), m_{e}\right]++i\left(\chi_{L} \bar{\epsilon}_{R}-\epsilon_{L} \bar{\chi}_{R}\right) m_{o}^{(-)}-i m_{o}^{(-)}\left(\chi_{R} \bar{\epsilon}_{L}-\epsilon_{R} \bar{\chi}_{L}\right) .
\end{aligned}
$$

The commutation relations between the homogeneous and inhomogeneous supersymmetry transformations are similarly written as

$$
\begin{aligned}
{\left[\delta_{\chi}^{(1)}, \delta_{\epsilon}^{(2)}\right] m_{e} } & =-i\left(\chi_{L} \bar{\epsilon}_{R}-\epsilon_{L} \bar{\chi}_{R}+\chi_{R} \bar{\epsilon}_{L}-\epsilon_{R} \bar{\chi}_{L}\right), \\
{\left[\delta_{\chi}^{(1)}, \delta_{\epsilon}^{(2)}\right] m_{o}^{(+)} } & =-i\left(\chi_{R} \bar{\epsilon}_{R}-\epsilon_{R} \bar{\chi}_{R}\right), \\
{\left[\delta_{\chi}^{(1)}, \delta_{\epsilon}^{(2)}\right] m_{o}^{(-)} } & =-i\left(\chi_{L} \bar{\epsilon}_{L}-\epsilon_{L} \bar{\chi}_{L}\right) .
\end{aligned}
$$

These commutation relations (2.38) show that constant shifts of the $+(-)$ fields are generated by the right(left)-handed supersymmetries. If we neglect the $m_{e}$ fields, the two sectors $\left(m_{o}^{(+)}\right.$ and $\left.\psi_{R}\right)$ and $\left(m_{o}^{(-)}\right.$and $\left.\psi_{L}\right)$ are completely decoupled. If we can successfully integrate out $m_{e}$, $m_{o}^{(-)}$and $\psi_{L}$ fields, we expect to obtain a IIB-like matrix model. As we see from (2.34), if we simply neglect these fields, the right-handed homogeneous supersymmetry transformations for the remaining fields $\left(m_{o}^{(+)}, \psi_{R}\right)$ become

$$
\delta_{\chi_{R}}^{(1)} m_{o}^{(+)}=i\left(\chi_{R} \bar{\psi}_{R}-\psi_{R} \bar{\chi}_{R}\right), \quad \delta_{\chi_{R}}^{(1)} \psi_{R}=-\left\langle m_{e}\right\rangle \chi_{R}
$$


Here $\left\langle m_{e}\right\rangle$ should be understood as the vacuum expectation value expressed in terms of $m_{o}^{(+)}$. Hence if $C_{\mu \nu}$ field in $\left\langle m_{e}\right\rangle$ is replaced by $\left[A_{\mu}^{(+)}, A_{\nu}^{(+)}\right]$, the transformation law can be identified with the homogeneous supersymmetry in IIB matrix model.

Let us look at these transformations explicitly for the rank 1 field $A_{\mu}^{( \pm)}$. Under the homogeneous supersymmetry transformation, they transform as

$$
\delta_{\chi}^{(1)} A_{\mu}^{(+)}=\frac{i}{8} \bar{\chi}_{R} \Gamma_{\mu} \psi_{R}, \quad \delta_{\chi}^{(1)} A_{\mu}^{(-)}=\frac{i}{8} \bar{\chi}_{L} \Gamma_{\mu} \psi_{L}
$$

We next consider the commutation relations among supersymmetry acting on $A_{\mu}^{( \pm)}$fields. Extracting the specific chirality of the supersymmetry parameters, the commutators become

$$
\begin{array}{ll}
{\left[\delta_{\chi_{R}}^{(1)}, \delta_{\epsilon_{R}}^{(2)}\right] A_{\mu}^{(-)}=0,} & {\left[\delta_{\chi_{R}}^{(1)}, \delta_{\epsilon_{R}}^{(2)}\right] A_{\mu}^{(+)}=\frac{i}{8} \bar{\epsilon}_{R} \Gamma_{\mu} \chi_{R},} \\
{\left[\delta_{\chi_{L}}^{(1)}, \delta_{\epsilon_{L}}^{(2)}\right] A_{\mu}^{(-)}=\frac{i}{8} \bar{\epsilon}_{L} \Gamma_{\mu} \chi_{L},} & {\left[\delta_{\chi_{L}}^{(1)}, \delta_{\epsilon_{L}}^{(2)}\right] A_{\mu}^{(+)}=0 .}
\end{array}
$$

This is consistent with the above identification of pairs: $A_{\mu}^{(-)}\left(A_{\mu}^{(+)}\right)$field is paired with the left (right) chirality. Commutators of two supersymmetry parameters with different chiralities vanish when they act on $A_{\mu}^{( \pm)}$:

$$
\left[\delta_{\chi_{L}}^{(1)}, \delta_{\epsilon_{R}}^{(2)}\right] A_{\mu}^{( \pm)}=\left[\delta_{\chi_{R}}^{(1)}, \delta_{\epsilon_{L}}^{(2)}\right] A_{\mu}^{( \pm)}=0
$$

We then look at the commutation relations between homogeneous supersymmetries. We are interested in which generators of $s p(32, R)$ rotations appear in the commutator. The commutators between the same chirality

$$
\left[\delta_{\chi_{R}}^{(1)}, \delta_{\epsilon_{R}}^{(1)}\right] A_{\mu}^{(+)}=\frac{i}{8}\left(\bar{\chi}_{R}\left[m, \Gamma_{\mu}\right] \epsilon_{R}\right)
$$

survive only for the fields of even rank $m_{e}\left(W, C_{i_{1} i_{2}}\right.$ and $\left.H_{i_{1} \cdots i_{4}}\right)$ in $m$ in the r.h.s. Since these fields are integrated out at last, we do not mind the appearance. On the other hand, commutators between different chiralities

$$
\left[\delta_{\chi_{L}}^{(1)}, \delta_{\epsilon_{R}}^{(1)}\right] A_{\mu}^{(+)}=\frac{i}{16}\left(\bar{\chi}_{L} m \Gamma_{\mu} \epsilon_{R}+\bar{\epsilon}_{R} \Gamma_{\mu} m \chi_{L}\right)
$$

survive for odd rank fields, $A_{\mu}^{(+)}$and $I_{\mu_{1} \cdots \mu_{5}}^{(+)}$, and contains the field $A_{\mu}^{(+)}$itself as

$$
\left[\delta_{\chi_{L}}^{(1)}, \delta_{\epsilon_{R}}^{(1)}\right] A_{\mu}^{(+)}=\frac{-i}{8} \bar{\chi}_{L} A_{\nu}^{(+)} \Gamma_{\mu}^{\nu} \epsilon_{R}+\cdots
$$

The r.h.s. is generated by $S O(9,1)$ rotation. Hence, if we interpret the eigenvalue distribution of $A_{\mu}^{(+)}$or $A_{\mu}^{(-)}$as space-time extension, we need to perform $S O(9,1)$ rotation to obtain 
the correct space-time supersymmetry simultaneously with supersymmetries. In this sense, $S O(9,1)$ symmetry should be more appropriately considered as a kind of gauge symmetry.

The above symmetry arguments support our expectation that the $\operatorname{ssp}(1 \mid 32, R)$ matrix model becomes IIB matrix model after integrating out some fields. However, there are no terms in the action consistent with the pairing expected from the symmetry arguments (2.35). In the next subsection we discuss a possibility and also a difficulty to obtain the correct coupling between fermion and boson by integrating out unnecessary fields.

\subsection{Integrating out $m_{e}, m_{o}^{(-)}$and $\psi_{L}$ fields}

In order to show that the correct coupling between $\psi_{R}$ and $A_{\mu}^{(+)}$can be generated, we need to integrate out the unnecessary fields $\left(m_{e}, m_{o}^{(-)}\right.$and $\left.\psi_{L}\right)$. For this purpose, these unnecessary fields need to have quadratic terms which may be generated by giving vacuum expectation values to some fields. The action (2.32) indicates that, if $W$ acquires a vacuum expectation value, quadratic terms do not appear for $m_{e}$ fields. On the other hand, if $A_{\mu}^{(+)}$acquires VEV such as the noncommutative $\hat{p}_{\mu}$, all the unnecessary fields can get quadratic terms. Hence in the following we expand $A_{\mu}^{(+)}$fields around the noncommutative classical background

$$
A_{\mu}^{(+)}=\hat{p}_{\mu}+a_{\mu}^{(+)}
$$

where $\hat{p}_{\mu}$ satisfy $\left[\hat{p}_{\mu}, \hat{p}_{\mu}\right]=i \beta_{\mu \nu}$ and each $\beta_{\mu \nu}$ is a $c$-number. Applying to the action the mapping rule from matrices to functions (briefly reviewed in the next section), we obtain the following action. In the following expression, all products are the so-called star products and $\operatorname{Tr}_{N \times N}$ should be understood as an integral over noncommutative space. The quadratic terms are given by

$$
\begin{aligned}
I_{b}^{(2)} & =\frac{1}{g^{2}} \operatorname{Tr}_{N \times N}\left(-96\left(\partial_{\mu_{1}} A_{\mu_{2}}^{(-)}\right) C^{\mu_{1} \mu_{2}}+96\left(\partial_{\mu} W\right) A^{(-) \mu}+4\left(\partial_{\mu_{1}} H_{\mu_{2} \cdots \mu_{5}}\right) I^{(-) \mu_{1} \cdots \mu_{5}}\right. \\
I_{f}^{(2)} & =\frac{1}{g^{2}} \operatorname{Tr}_{N \times N}\left(-3 i \bar{\psi}_{L} \Gamma^{\mu} \partial_{\mu} \psi_{L}\right) .
\end{aligned}
$$

The cubic interaction terms are given by

$$
\begin{aligned}
I_{b}^{(3)} & =\frac{i}{g^{2}} \operatorname{Tr}_{N \times N}\left(-96\left[a_{\mu_{1}}^{(+)}, A_{\mu_{2}}^{(-)}\right] C^{\mu_{1} \mu_{2}}-96 W\left[a^{(+) \mu}, A_{\mu}^{(-)}\right]+\frac{4}{5} W\left[I_{\mu_{1} \cdots \mu_{5}}^{(+)}, I^{(-) \mu_{1} \cdots \mu_{5}}\right]\right. \\
& +4\left(\left[a_{\mu_{1}}^{(+)}, H_{\mu_{2} \cdots \mu_{5}}\right] I^{(-) \mu_{1} \cdots \mu_{5}}-\left[A_{\mu_{1}}^{(-)}, H_{\mu_{2} \cdots \mu_{5}}\right] I^{(+) \mu_{1} \cdots \mu_{5}}\right)-8 C_{\mu_{1} \mu_{2}}\left[I^{(+) \mu_{1}}{ }_{\mu_{3} \cdots \mu_{6}}, I^{(-) \mu_{2} \cdots \mu_{6}}\right] \\
& +\frac{8}{3} H_{\mu_{1} \mu_{2}}^{\nu \lambda}\left(\left[I^{(+)}{ }_{\nu \lambda \mu_{3} \mu_{4} \mu_{5}}, I^{(-) \mu_{1} \cdots \mu_{5}}\right]-\left[I^{(-)}{ }_{\nu \lambda \mu_{3} \mu_{4} \mu_{5}}, I^{(+) \mu_{1} \cdots \mu_{5}}\right]\right) \\
& +32\left[C_{\mu_{2}}^{\mu_{1}}, C_{\mu_{1} \mu_{3}}\right] C^{\mu_{2} \mu_{3}}-16 C_{\mu_{1} \mu_{2}}\left[H_{\mu_{3} \mu_{4} \mu_{5}}^{\mu_{1}}, H^{\mu_{2} \cdots \mu_{5}}\right]
\end{aligned}
$$




$$
\begin{aligned}
& \left.+\frac{1}{27} H_{\mu_{1} \cdots \mu_{4}}\left[H_{\mu_{5} \cdots \mu_{7}}^{\nu}, H_{\nu \mu_{8} \mu_{9} \mu_{10}}\right] \epsilon^{\mu_{1} \cdots \mu_{10 \sharp}}\right) \\
I_{f}^{(3)} & =\frac{i}{g^{2}} \operatorname{Tr}_{N \times N}\left(-3 i\left(-\bar{\psi}_{L}\left[W, \psi_{R}\right]+\bar{\psi}_{R}\left[W, \psi_{L}\right]\right)-3 i\left(\bar{\psi}_{L} \Gamma^{\mu}\left[a_{\mu}^{(+)}, \psi_{L}\right]+\bar{\psi}_{R} \Gamma^{\mu}\left[A_{\mu}^{(-)}, \psi_{R}\right]\right)\right. \\
& -\frac{3 i}{2 !}\left(\bar{\psi}_{L} \Gamma^{\mu_{1} \mu_{2}}\left[C_{\mu_{1} \mu_{2}}, \psi_{R}\right]+\bar{\psi}_{R} \Gamma^{\mu_{1} \mu_{2}}\left[C_{\mu_{1} \mu_{2}}, \psi_{L}\right]\right) \\
& -\frac{3 i}{4 !}\left(-\bar{\psi}_{L} \Gamma^{\mu_{1} \mu_{2} \mu_{3} \mu_{4}}\left[H_{\mu_{1} \mu_{2} \mu_{3} \mu_{4}}, \psi_{R}\right]+\bar{\psi}_{R} \Gamma^{\mu_{1} \mu_{2} \mu_{3} \mu_{4}}\left[H_{\mu_{1} \mu_{2} \mu_{3} \mu_{4}}, \psi_{L}\right]\right) \\
& \left.-\frac{3 i}{5 !}\left(2 \bar{\psi}_{L} \Gamma^{\mu_{1} \mu_{2} \mu_{3} \mu_{4} \mu_{5}}\left[I_{\mu_{1} \mu_{2} \mu_{3} \mu_{4} \mu_{5}}^{(+)}, \psi_{L}\right]+2 \bar{\psi}_{R} \Gamma^{\mu_{1} \mu_{2} \mu_{3} \mu_{4} \mu_{5}}\left[I_{\mu_{1} \mu_{2} \mu_{3} \mu_{4} \mu_{5}}^{(-)}, \psi_{R}\right]\right)\right)
\end{aligned}
$$

We draw some typical vertices in fig. 11.

\section{Vertices}

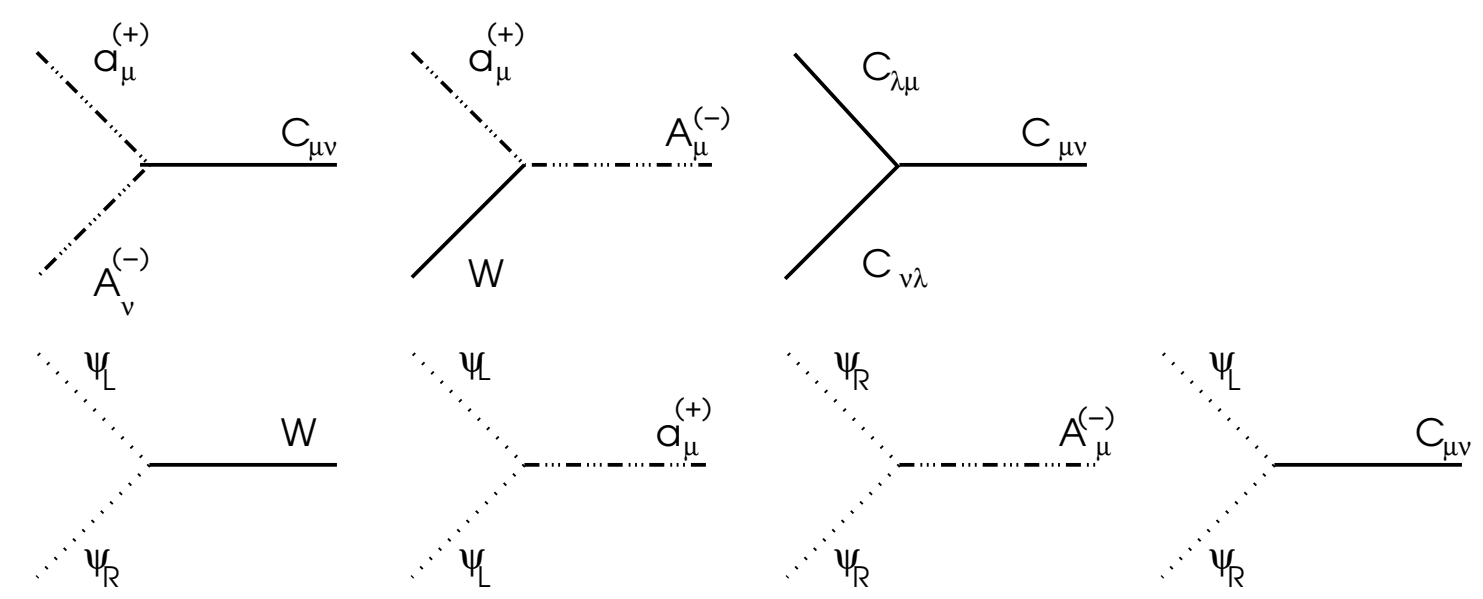

Figure 1: Typical vertices of the $\operatorname{osp}(1 \mid 32, R)$ matrix model

If we neglect the interaction terms first, an integration over $C_{\mu \nu}$ field gives a constraint on $A^{(-)}$as

$$
\partial_{\mu} A_{\nu}^{(-)}-\partial_{\nu} A_{\mu}^{(-)}=0
$$

and we can solve it in terms of a scalar as $A_{\mu}^{(-)}=\partial_{\mu} \lambda$. Inserting this into the quadratic term, we have a propagator connecting two scalars, $\lambda$ and $W$. Then we can integrate unnecessary fields $A_{\mu}^{(-)}, W I^{(-)}, H$ and $\psi_{L}$. An issue here is whether we can generate the IIB-like terms such as $\operatorname{Tr}_{N \times N}\left[A_{\mu}^{(+)}, A_{\nu}^{(+)}\right]^{2}$ or $\operatorname{Tr}_{N \times N} \bar{\psi}_{R} \Gamma^{\mu}\left[A_{\mu}^{(+)}, \psi_{R}\right]$. $U(N)$ gauge symmetry assures the existence of these terms if we can show that quadratic kinetic terms for $a_{\mu}^{(+)}$and $\psi_{R}$, that is, $\left(\partial_{\mu} a_{\nu}^{(+)}-\partial_{\nu} a_{\mu}^{(+)}\right)^{2}$ and $\bar{\psi}_{R} \Gamma^{\mu} \partial_{\mu} \psi_{R}$, are generated. First, the kinetic term for $a_{\mu}^{(+)}$is easily generated by integrating out $\psi_{L}$ as in fig. 2, since there is a vertex $\bar{\psi}_{L} \Gamma^{\mu}\left[a_{\mu}^{(+)}, \psi_{L}\right]$. 


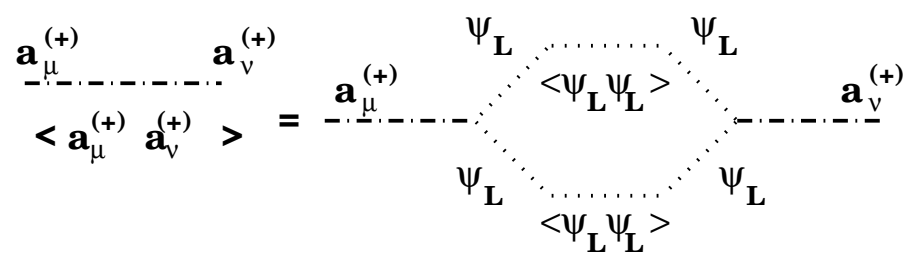

Figure 2: A propagator $\left\langle a_{\mu}^{(+)} a_{\nu}^{(+)}\right\rangle$is induced by one-loop effect.

The kinetic term for $\psi_{R}$ is more difficult to generate. As is seen from fig. 3, one way to generate such a term is to connect two $\bar{\psi}_{L}\left[W, \psi_{R}\right]$ vertices by propagators of $\psi_{L}$ and $W$.

\section{Induced propagator}

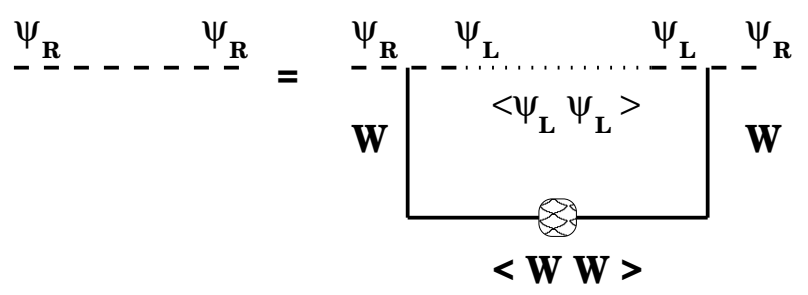

Figure 3: A propagator for $\psi_{R}$ is induced if $W$ can acquire a propagator.

Propagators for $W$ and $\lambda$ fields cannot be generated perturbatively as we prove in Appendix B. But since there is no symmetry prohibiting such terms it does not exclude a nonperturbative generation. The existence of the propagator connecting these two scalar fields rather indicate that both of these two propagators can be generated self-consistently. We do not discuss more details here, but it is probable that $W$ acquires a propagator and the above mentioned kinetic term for $\psi_{R}$ will be also generated. In this way, we expect that IIB matrix model is induced from osp $(1 \mid 32, R)$ model.

\section{Diffeomorphism in noncommutative Yang-Mills}

In this section, we first review how noncommutative Yang-Mills is obtained from matrix models and then investigate the special properties regarding the local gauge transformations in noncommutative background. Especially we study special types of gauge transformations which can be interpreted as local coordinate transformations.

First we give a brief review on a matrix model description of noncommutative field 
theories. The noncommutative background $\hat{x}$ satisfying

$$
\left[\hat{x}^{\mu}, \hat{x}^{\nu}\right]=-i \theta^{\mu \nu}
$$

with a c-number $\theta^{\mu \nu}$ is a classical solution of IIB matrix model. We assume the rank of $\theta^{\mu \nu}$ to be $\tilde{d}$ and define its inverse $\beta_{\mu \nu}$ in $\tilde{d}$-dimensional subspace. This expression is formal and only valid for infinite $N$. For finite $N$, see papers [16]. $\hat{x}^{\mu}$ satisfy the canonical commutation relations and they span the $\tilde{d}$-dimensional phase space. Therefore the momentum operators are proportional to the coordinates as

$$
\hat{p}_{\mu}=\beta_{\mu \nu} \hat{x}^{\nu} .
$$

The semiclassical correspondence shows that the volume of the phase space (measured in the coordinate space of $\left.x^{\mu}\right)$ is $V=N(2 \pi)^{\tilde{d} / 2} \sqrt{\operatorname{det} \theta}$. We expand the bosonic matrices $A^{\mu}$ around $\hat{x}^{\mu}=\theta^{\mu \nu} \hat{p}_{\nu}$ as

$$
A^{\mu}=\theta^{\mu \nu}\left(\hat{p}_{\nu}+\tilde{a}_{\nu}\right) .
$$

If we assume that all fields can be expanded in terms of noncommutative plane wave $\exp (i k$. $\hat{x}$ ), we obtain a map from a matrix

$$
\hat{a}=\sum_{k} \tilde{a}(k) \exp (i k \cdot \hat{x})
$$

to a function

$$
a(x)=\sum_{k} \tilde{a}(k) \exp (i k \cdot x)
$$

in the $\tilde{d}$-dimensional noncommutative plane. By this construction, a product of matrices is mapped to the $\star$ product of functions

$$
\begin{aligned}
\hat{a} \hat{b} & \rightarrow a(x) \star b(x) \\
a(x) \star b(x) & \left.\equiv \exp \left(\frac{\theta^{\mu \nu}}{2 i} \frac{\partial^{2}}{\partial \xi^{\mu} \partial \eta^{\nu}}\right) a(x+\xi) b(x+\eta)\right|_{\xi=\eta=0}
\end{aligned}
$$

and the operation $\operatorname{Tr}$ over matrices can be exactly mapped onto the integration over functions as

$$
\operatorname{Tr}[\hat{a}]=\sqrt{\operatorname{det} \beta}\left(\frac{1}{2 \pi}\right)^{\frac{\tilde{d}}{2}} \int d^{\tilde{d}} x a(x) .
$$

The reduced model can be shown to be equivalent to noncommutative Yang-Mills by the following map from matrices onto functions

$$
\begin{aligned}
\hat{a} & \rightarrow a(x), \\
\hat{a} \hat{b} & \rightarrow a(x) \star b(x), \\
T r & \rightarrow \sqrt{\operatorname{det} \beta}\left(\frac{1}{2 \pi}\right)^{\frac{\tilde{d}}{2}} \int d^{\tilde{d}} x .
\end{aligned}
$$


Applying the rule eq.(3.8), we can obtain $U(1)$ gauge theory on $\tilde{d}$-dimensional noncommutative space. (Noncommutative $U(m)$ gauge theory can be similarly obtained by expanding around $x^{\mu} \otimes 1_{m}$.)

The extension of $\hat{x}^{\mu}$ can be interpreted as the space-time and the space-time translation is realized by the following unitary operator:

$$
\exp (i \hat{p} \cdot \epsilon) \hat{x}^{\mu} \exp (-i \hat{p} \cdot \epsilon)=\hat{x}^{\mu}+\epsilon^{\mu} .
$$

It is amusing that the translation in the noncommutative space is realized by $U(N)$ gauge transformations in matrix models. This realization has been known as Parisi prescription in the old reduced models [17] and reinvestigated [18] [19] from the noncommutative point of view to study extended gauge invariant operators (open Wilson lines). The local gauge symmetry of noncommutative Yang-Mills is originated in the invariance under $U(N)$ invariance of IIB matrix model

$$
A_{\mu} \rightarrow U A_{\mu} U^{\dagger} .
$$

Indeed, if we expand $U=\exp (i \hat{\lambda})$ and parameterize $\hat{\lambda}$ as

$$
\hat{\lambda}=\sum_{k} \tilde{\lambda}(k) \exp (i k \cdot \hat{x}),
$$

we find that the fluctuating field of $A_{\mu}$ around the fixed noncommutative background transforms as

$$
\hat{a}_{\mu} \rightarrow \hat{a}_{\mu}+i\left[\hat{p}_{\mu}, \hat{\lambda}\right]-i\left[\hat{a}_{\mu}, \hat{\lambda}\right] .
$$

After mapping the transformation onto functions, we have

$$
\begin{aligned}
& a_{\alpha}(x) \rightarrow a_{\alpha}(x)+\frac{\partial}{\partial x^{\alpha}} \lambda(x)-i\left[a_{\alpha}(x), \lambda(x)\right]_{\star}, \\
& a_{i} \rightarrow a_{i}-i\left[a_{i}(x), \lambda(x)\right]_{\star}, \\
& \psi \rightarrow \psi-i[\psi(x), \lambda(x)]_{\star},
\end{aligned}
$$

where $1 \leq \alpha \leq \tilde{d}$ and $i>\tilde{d}$. If we take $\lambda$ as in (3.9),

$$
\hat{\lambda}=\epsilon^{\alpha} \hat{p}_{\alpha},
$$

the transformations (3.13) become translation in the noncommutative space up to a constant shift of the gauge field:

$$
\begin{aligned}
& a_{\alpha}(x) \rightarrow a_{\alpha}(x)-\beta_{\alpha \beta} \epsilon^{\beta}+\epsilon^{\beta} \partial_{\beta} a_{\alpha}(x), \\
& a_{i}(x) \rightarrow a_{i}(x)+\epsilon^{\beta} \partial_{\beta} a_{i}(x), \\
& \psi(x) \rightarrow \psi(x)+\epsilon^{\beta} \partial_{\beta} \psi(x) .
\end{aligned}
$$


As the above example shows, local gauge symmetries in noncommutative gauge theories are very different from those in the ordinary gauge theories and even space-time translation is generated. Hence all gauge invariant operators are invariant under the space-time translation and should be constructed by integrating over space-time, which is reminiscent of the theory of gravity. We are, therefore, tempted to generalize the above discussion to local transformations. The reason why the gauge transformations in noncommutative space-time are much larger than those in commutative space is that gauge transformation parameters $\lambda$ contain not only ordinary functions but differential operators in the semiclassical limit in the following sense. Functions in noncommutative space are expanded in terms of noncommutative plane waves as in (3.11). For finite $N$, the momenta of plane waves take values of

$$
k_{n}=\sqrt{2 \pi \beta} N^{-1 / \tilde{d}} n
$$

where $n=0,1, \cdots, N^{2 / \tilde{d}}$. This can be seen from the explicit construction of the plane waves in terms of 't Hooft matrices $U$ and $V$ satisfying $U V=\exp (2 \pi i / N) V U$. The number of independent plane waves is $N^{2}$, which is the same as the number of degrees of freedom of a hermitian matrix. Since the natural cut off scale in the noncommutative plane (1.7) is given by $l_{0}=\sqrt{2 \pi \theta}=\sqrt{2 \pi / \beta}$, the natural cut off of momenta should be $2 \pi / l_{0}=\sqrt{2 \pi \beta}$. However, some plane waves with momenta (3.16) exceed this natural bound and they become very nonlocal objects since such high momentum plane waves generate translation in space with $l_{0} N^{-1 / \tilde{d}} n$. Hence only $N$ out of $N^{2}$ plane waves whose momenta are smaller than $\sqrt{2 \pi \beta}$ can be interpreted as ordinary plane waves in the semiclassical limit and others should be interpreted as differential operators that can generate translation in noncommutative direction in space-time. From a matrix model point of view, such nonlocal waves correspond to off-diagonal elements while local ones to diagonal elements.

Now let us consider the gauge transformations related to local coordinate transformations. A natural generalization of the global translation (3.14) will be

$$
\lambda=\frac{1}{2}\left(\hat{p}_{\alpha} \hat{\epsilon}^{\alpha}+\hat{\epsilon}^{\alpha} \hat{p}_{\alpha}\right)
$$

or, if we want to include both of the gauge transformations and local coordinate transformations in the semiclassical limit, we can expand $\lambda$ as

$$
\lambda=\hat{\lambda}_{0}+\frac{1}{2}\left(\hat{p}_{\alpha} \hat{\epsilon}^{\alpha}+\hat{\epsilon}^{\alpha} \hat{p}_{\alpha}\right)
$$


Similarly we expand bosonic matrices as

$$
\tilde{A}_{\mu}=\beta_{\mu \nu} A^{\nu}=\tilde{a}_{\mu}+\frac{1}{2}\left(\hat{p}_{\alpha} \hat{e}_{\mu}^{\alpha}+\hat{e}_{\mu}^{\alpha} \hat{p}_{\alpha}\right),
$$

and assume that the field $\hat{e}_{\mu}^{\alpha}$ is close to $\delta_{\mu}^{\alpha}$ :

$$
\hat{e}_{\mu}^{\alpha}=\delta_{\mu}^{\alpha}+h_{\mu}^{\alpha} .
$$

This is a natural generalization of the expansion (3.3). Applying the unitary transformation generated by (3.17) to the bosonic field expanded as above, we have the following transformation law:

$$
\begin{aligned}
\delta h_{\mu}^{\alpha}(x) & =-e_{\mu}^{\beta}(x) \partial_{\beta} \epsilon^{\alpha}+\epsilon^{\beta} \partial_{\beta} h_{\mu}^{\alpha}, \\
\delta a_{\mu}(x) & =\epsilon^{\beta} \partial_{\beta} a_{\mu}(x) .
\end{aligned}
$$

Here we have assumed that all of $\epsilon^{\alpha}, a_{\mu}$ and $e_{\mu}^{\alpha}$ are slowly varying and dropped higher derivative terms. In addition to the transformations of the fields, we need to transform the background as

$$
\delta \hat{p}_{\alpha}=\beta_{\alpha \beta} \hat{\epsilon}^{\beta}
$$

or $\delta \hat{x}^{\mu}=\epsilon^{\mu}$ in terms of $\hat{x}^{\mu}$. Accordingly, the commutation relations between $\hat{p}_{\mu}$ change as

$$
\left[\hat{p}_{\alpha}+\beta_{\alpha \beta} \hat{\epsilon}^{\beta}, \hat{p}_{\alpha^{\prime}}+\beta_{\alpha^{\prime} \beta^{\prime}} \hat{\epsilon}^{\beta^{\prime}}\right]=i\left(\beta_{\alpha \alpha^{\prime}}+\beta_{\beta \alpha^{\prime}} \partial_{\alpha} \epsilon^{\beta}+\beta_{\alpha \beta} \partial_{\alpha^{\prime}} \epsilon^{\beta}\right) .
$$

Therefore the shift of the background can be interpreted as the transformation of $\beta_{\alpha \alpha^{\prime}}$.

The transformation (3.21) indicates that local coordinate transformations can be realized by gauge transformations in noncommutative space. But as the transformations (3.21) show, the gauge field and the fermion field transform as a scalar and $h_{\mu}{ }^{\alpha}$ as a vector field. In other words, the $S O(9,1)$ index has nothing to do with this coordinate transformation as it should be since $S U(N)$ transformations and $S O(9,1)$ transformations are independent from the beginning. IIB matrix model is not explicitly invariant under local $S O(9,1)$. Local Lorentz transformations might be realized in a complicated way in IIB matrix model and we expect that there is an extended model with obvious local Lorentz symmetry, which becomes IIB matrix model after gauge fixing. In the next section, we search for such models.

\section{Gauged matrix models}

In this section we investigate another type of matrix models with larger local symmetries. The model studied in section 2 has an extension of $S O(9,1)$ symmetry, that is, $\operatorname{OSp}(1 \mid 32, R)$ 
symmetry. But this symmetry is decoupled from $U(N)$ gauge symmetry. As we have seen in section 3, space-time is realized as eigenvalues of bosonic matrices and consequently some $U(N)$ symmetry is identified with space-time translation. Hence if local Lorentz symmetry exists it should be $U(N)$ dependent $S O(9,1)$ symmetry and we need to unify decoupled $S O(9,1)$ and $U(N)$ invariance in IIB matrix model.

Let us first try to gauge the global $S O(9,1)$ symmetry. A convenient way to write $S O(9,1)$ is to use $\gamma$-matrices. Defining

$$
m=\Gamma^{\mu} A_{\mu}, \quad h=\frac{1}{2} \zeta_{\mu \nu} \Gamma^{\mu \nu},
$$

$S O(9,1)$ rotations of $A_{\mu}$ and $\psi$ are given by

$$
\delta m=[h, m], \quad \delta \psi=h \psi, \quad \delta \bar{\psi}=-\bar{\psi} h .
$$

The rotation angle $\zeta_{\mu \nu}$ is a $c$-number. One way to gauge global symmetries in matrix models is to make transformation parameters $U(N)$ dependent. A big difference here from local gauge symmetries in ordinary commutative space-time is that $U(N)$-dependent matrices are generally not commutative while $x$-dependent local parameters are of course commutative. Therefore, the algebra does not close within the original transformations. In our case of $S O(9,1)$, since

$$
\left[\hat{h}, \hat{h}^{\prime}\right]=\left[\frac{1}{2} \hat{\zeta}_{\mu \nu} \Gamma^{\mu \nu}, \frac{1}{2} \hat{\zeta}_{\mu^{\prime} \nu^{\prime}} \Gamma^{\mu^{\prime} \nu^{\prime}}\right]=\frac{1}{8}\left(\left[\Gamma^{\mu \nu}, \Gamma^{\mu^{\prime} \nu^{\prime}}\right]\left\{\hat{\zeta}_{\mu \nu}, \hat{\zeta}_{\mu^{\prime} \nu^{\prime}}\right\}+\left\{\Gamma^{\mu \nu}, \Gamma^{\mu^{\prime} \nu^{\prime}}\right\}\left[\hat{\zeta}_{\mu \nu}, \hat{\zeta}_{\mu^{\prime} \nu^{\prime}}\right]\right),
$$

and the commutator between $\hat{\zeta}_{\mu \nu}$ does not vanish, we need to include transformations generated by the anti-commutators of $\Gamma^{\mu \nu}$, that is, 1 and $\Gamma^{\mu_{1} \mu_{2} \mu_{3} \mu_{4}}$. Repeating this procedure, the algebra finally closes in the $\gamma$-matrices with even rank, $1, \Gamma^{\sharp}, \Gamma^{\mu \nu}, \Gamma^{\mu \nu \sharp}, \Gamma^{\mu_{1} \mu_{2} \mu_{3} \mu_{4}}$ and $\Gamma^{\mu_{1} \mu_{2} \mu_{3} \mu_{4} \sharp}$. There are 512 bosonic generators. The coefficients $\zeta_{\mu \nu}$ must be extended to complex matrices. Since these transformations can be restricted to chiral sectors of fermions, we can obtain closed gauged algebra acting on Weyl fermions of IIB type. Generalizing this bosonic algebra by including supersymmetries, we obtain $g l(1 \mid 16, C)$ super Lie algebra. As far as the algebras are concerned, $g l(1 \mid 16, C)$ is a minimal gauged extension of $s o(9,1)$. As for dynamical fields, if we start from a vector boson with rank $1 \gamma$-matrix, $g l(16, C)$ bosonic transformations generate fields with other odd rank $\gamma$-matrices and we have to include $A_{\mu_{1} \mu_{2} \mu_{3}}$ and $A_{\mu_{1} \cdots \mu_{5}}$ in addition to $A_{\mu}$. There are 256 bosonic fields. A model based on this $g l(1 \mid 16, C)$ super Lie algebra is an interesting possibility, but it turns out difficult to find an invariant action. In the following we instead investigate a model with local osp $(1 \mid 32, R)$ 
gauge symmetry. That is, we demand that the model should be invariant under $U(N)$ dependent $\operatorname{osp}(1 \mid 32, R)$ symmetry. This model has larger symmetries and more fields than the $g l(16, C)$ model, but the invariant action can be easily constructed in terms of supermatrices as we show below. We must extend the chiral fermions to include both chiralities. We also have to extend the bosonic degrees of freedom $m$ by including fields with all ranks in 11 dimensions.

In constructing an invariant action of the gauged matrix model, it is generally difficult to keep both the gauge symmetries and invariance under a constant shift of fields. If the fields transform as in (4.2) after gauging, the action of the type $\operatorname{Tr}_{N \times N}(\bar{\psi} m \psi)$ or $\operatorname{Tr}_{N \times N}\left(m^{3}\right)$ are invariant. However, the action such as $\operatorname{Tr}_{N \times N}\left(\bar{\psi} \Gamma^{\mu}\left[A_{\mu}, \psi\right]\right)$ is not invariant under gauge transformations and it is difficult to keep both invariances. In this paper we abandon the latter invariance and consider the action

$$
I=\frac{1}{g^{2}} \operatorname{Tr}_{N \times N} \operatorname{Str}_{33 \times 33}\left(M^{3}\right) .
$$

This action was also proposed by Smolin. We call it a gauged model because it is invariant under local $\operatorname{osp}(1 \mid 32, R)$ symmetry, that is, a tensor product of two gauge symmetries $o s p(1 \mid 32, R)$ and $u(N)$. Instead of this enhancement of the symmetries, this action is not invariant under a constant shift of field. This looks troubling since, as we saw in section 2, commutators between the homogeneous and the inhomogeneous supersymmetries generate a space-time translation, a constant shift of bosonic field, and if we lose inhomogeneous translational invariance of bosons and fermions we may also lose space-time interpretation of supersymmetries. However, this problem can be resolved by identifying some generators of $\operatorname{osp}(1 \mid 32, R)$ (or its extension $u(1 \mid 16,16)$ ) with space-time translation generators using the Wigner-Inönü contraction.

There are two ways to gauge the $\operatorname{ssp}(1 \mid 32, R)$ model. One way proposed by Smolin is to use $u(1 \mid 16,16)$ super Lie algebra, a complexification of $\operatorname{osp}(1 \mid 32, R)$. He conjectured that this gauged model describes loop quantum gravity [15]. Another way to gauge is to use $g l(1 \mid 32, R)$ super Lie algebra, an analytic continuation of $u(1 \mid 16,16)$. We next see the definitions of these super Lie algebras and also see why they are gauged symmetries of the global $\operatorname{osp}(1 \mid 32, R)$. 


\subsection{Definitions of $u(1 \mid 16,16)$ and $g l(1 \mid 32, R)$}

An element $M$ of $u(1 \mid 16,16)$ super Lie algebra satisfies

$$
M^{\dagger} G+G M=0 \text { for } G=\left(\begin{array}{cc}
\Gamma^{0} & 0 \\
0 & i
\end{array}\right) .
$$

The reality condition is not imposed in this case. The above definition restricts the $33 \times 33$ matrix form of $M$ as

$$
M=\left(\begin{array}{ll}
m & \psi \\
i \bar{\psi} & v
\end{array}\right)
$$

where $v$ is pure imaginary, $\psi$ is a general complex spinor and $\bar{\psi}=\psi^{\dagger} \Gamma^{0}$. The bosonic part $m$ can be expanded in terms of 11-dimensional $\gamma$-matrices,

$$
\begin{aligned}
m & =u \mathbf{1}+u_{A_{1}} \Gamma^{A_{1}}+\frac{1}{2 !} u_{A_{1} A_{2}} \Gamma^{A_{1} A_{2}}+\frac{1}{3 !} u_{A_{1} A_{2} A_{3}} \Gamma^{A_{1} A_{2} A_{3}} \\
& +\frac{1}{4 !} u_{A_{1} \cdots A_{4}} \Gamma^{A_{1} \cdots A_{4}}+\frac{1}{5 !} u_{A_{1} \cdots A_{5}} \Gamma^{A_{1} \cdots A_{5}}
\end{aligned}
$$

where $u_{A_{1}}, u_{A_{1} A_{2}}$ and $u_{A_{1} \cdots A_{5}}$ are real, while $u, u_{A_{1} A_{2} A_{3}}$ and $u_{A_{1} \cdots A_{4}}$ are pure imaginary. Pure imaginary valued coefficients $u, u_{A_{1} A_{2} A_{3}}$ and $u_{A_{1} \cdots A_{4}}$ are new compared to osp $(1 \mid 32, R)$. Fermions are also doubled since we do not impose the Majorana condition. This matrix can be decomposed into two matrices

$$
M=H+A^{\prime}, \quad H=\left(\begin{array}{cc}
m_{h} & \psi_{h} \\
i \bar{\psi}_{h} & 0
\end{array}\right), \quad A^{\prime}=\left(\begin{array}{cc}
m_{a} & i \psi_{a} \\
\bar{\psi}_{a} & i v
\end{array}\right)
$$

where

$$
\begin{aligned}
& m_{h}=u_{A_{1}} \Gamma^{A_{1}}+\frac{1}{2 !} u_{A_{1} A_{2}} \Gamma^{A_{1} A_{2}}+\frac{1}{5 !} u_{A_{1} \cdots A_{5}} \Gamma^{A_{1} \cdots A_{5}}, \\
& m_{a}=u+\frac{1}{3 !} u_{A_{1} A_{2} A_{3}} \Gamma^{A_{1} A_{2} A_{3}}+\frac{1}{4 !} u_{A_{1} \cdots A_{4}} \Gamma^{A_{1} \cdots A_{4}}
\end{aligned}
$$

and $\psi_{h}$ and $\psi_{a}$ are real fermions. They satisfy the following relations

$$
{ }^{T} H G+G H=0, \quad{ }^{T} A^{\prime} G-G A^{\prime}=0 .
$$

The matrix $H$ forms $\operatorname{osp}(1 \mid 32, R)$ super Lie subalgebra of $u(1 \mid 16,16)$ algebra but $A^{\prime}$ does not form an algebra by themselves. We denote the former set of matrices by $\mathcal{H}$ and the latter by $\mathcal{A}^{\prime}$. Then the following commutation and anti-commutation structures are satisfied

$$
\begin{aligned}
& {[\mathcal{H}, \mathcal{H}] \in \mathcal{H},\left[\mathcal{H}, \mathcal{A}^{\prime}\right] \in \mathcal{A}^{\prime},\left[\mathcal{A}^{\prime}, \mathcal{A}^{\prime}\right] \in \mathcal{H},} \\
& \{\mathcal{H}, \mathcal{H}\} \in \mathcal{A}^{\prime},\left\{\mathcal{H}, \mathcal{A}^{\prime}\right\} \in \mathcal{H},\left\{\mathcal{A}^{\prime}, \mathcal{A}^{\prime}\right\} \in \mathcal{A}^{\prime}
\end{aligned}
$$


We can see that $A^{\prime}$ is another representation of $\operatorname{osp}(1 \mid 32, R)$.

The definition of $g l(1 \mid 32, R)$ super Lie algebra is simply given by the following form of $33 \times 33$ supermatrix

$$
M=\left(\begin{array}{cc}
m & \psi \\
i \bar{\phi} & v
\end{array}\right),
$$

where all components are real. The boson $m$ can be expanded similarly in terms of 11dimensional $\gamma$-matrices as in (4.7) but all the coefficients $u, \cdots, u_{A_{1} \cdots A_{5}}$ are real. Two fermions $\psi$ and $\phi$ are also real. This matrix is decomposed into two parts as

$$
M=H+A,
$$

where

$$
\begin{gathered}
H=\left(\begin{array}{cc}
m_{h} & \psi_{1} \\
i \bar{\psi}_{1} & 0
\end{array}\right), \\
A=\left(\begin{array}{cc}
m_{a} & \psi_{2} \\
-i \bar{\psi}_{2} & v
\end{array}\right) .
\end{gathered}
$$

Here we have defined $\psi_{i}$ by

$$
\psi=\psi_{1}+\psi_{2}, \quad \phi=\psi_{1}-\psi_{2}
$$

and $m_{h}$ and $m_{a}$ are given in (4.9) with real coefficients. $H$ is again an element of $\operatorname{ssp}(1 \mid 32, R)$ generators and $A$ is its representation.

These two super Lie algebras $u(1 \mid 16,16)$ and $g l(1 \mid 32, R)$ are related as follows. A matrix $M=H+A^{\prime}$ in $u(1 \mid 16,16)$ is mapped to a matrix in $g l(1 \mid 32, R)$ by $N=H+A$ where $A=i A^{\prime}$ and vice versa. Hence these two algebras are related by an analytic continuation.

We now promote each real element of matrices to an $N \times N$ hermitian matrix to make our model invariant under local $\operatorname{ssp}(1 \mid 32, R)$ symmetry. If we start from a set of $\operatorname{ssp}(1 \mid 32, R)$ matrices $\mathcal{H}$ and make a tensor product with $u(N)$, the algebra does not close within them because of the following relation:

$$
\begin{aligned}
{[(\mathcal{H} \otimes \mathbf{H}),(\mathcal{H} \otimes \mathbf{H})] } & =(\{\mathcal{H}, \mathcal{H}\} \otimes[\mathbf{H}, \mathbf{H}])+([\mathcal{H}, \mathcal{H}] \otimes\{\mathbf{H}, \mathbf{H}\}) \\
& =\left(\mathcal{A}^{\prime} \otimes \mathbf{A}\right)+(\mathcal{H} \otimes \mathbf{H})
\end{aligned}
$$

Here we have used (4.11) and denoted $\mathbf{H}$ and $\mathbf{A}$ as hermitian and anti-hermitian matrices. In order for the algebra to close, it is necessary to include $\mathcal{A}^{\prime} \otimes \mathbf{A}$. From the following relation

$$
\begin{aligned}
{\left[\left(\mathcal{A}^{\prime} \otimes \mathbf{A}\right),\left(\mathcal{A}^{\prime} \otimes \mathbf{A}\right)\right] } & =\left(\left\{\mathcal{A}^{\prime}, \mathcal{A}^{\prime}\right\} \otimes[\mathbf{A}, \mathbf{A}]\right)+\left(\left[\mathcal{A}^{\prime}, \mathcal{A}^{\prime}\right] \otimes\{\mathbf{A}, \mathbf{A}\}\right) \\
& =\left(\mathcal{A}^{\prime} \otimes \mathbf{A}\right)+(\mathcal{H} \otimes \mathbf{H})
\end{aligned}
$$


we can form a closed algebra by combining $(\mathcal{H} \otimes \mathbf{H})$ and $\left(\mathcal{A}^{\prime} \otimes \mathbf{A}\right)$ together. For $N=1$ case, $\mathbf{H}$ and $\mathbf{A}$ are replaced by 1 and $i$ respectively and it is nothing but $u(1 \mid 16,16)$ algebra we discussed before. This is a reason why we need to enlarge $\operatorname{osp}(1 \mid 32, R)$ to $u(1 \mid 16,16)$ for gauging the $\operatorname{osp}(1 \mid 32, R)$ symmetry.

Instead of promoting each element to a hermitian matrix, we can make a closed algebra by restricting them to real matrices. Since real matrices are closed under commutators and anti-commutations, it is clear that $(\mathcal{H}+\mathcal{A}) \otimes g l(N, R)=g l(1 \mid 32, R) \otimes g l(N, R)$ forms another closed algebra. In this case, we have to embed the space-time into real matrices instead of hermitian matrices.

\subsection{Action and symmetries}

The action we consider is

$$
\begin{aligned}
I & =\frac{1}{g^{2}} \operatorname{Tr}_{N \times N} \sum_{Q, R=1}^{33}\left(\left(\sum_{p=1}^{32} M_{p}{ }^{Q} M_{Q}{ }^{R} M_{R}{ }^{p}\right)-M_{33}{ }^{Q} M_{Q}{ }^{R} M_{R}{ }^{33}\right)=\frac{1}{g^{2}} \operatorname{Tr}_{N \times N}\left(\operatorname{Str}_{33 \times 33} M^{3}\right) \\
& =\frac{1}{g^{2}} \sum_{a, b, c=1}^{N^{2}} \operatorname{Str}_{33 \times 33}\left(M^{a} M^{b} M^{c}\right) \operatorname{Tr}_{N \times N}\left(t^{a} t^{b} t^{c}\right) .
\end{aligned}
$$

where $p=1, \cdots, 32$ and $Q, R=1, \cdots 33$. $M$ is a supermatrix belonging to $u(1 \mid 16,16)$ or $g l(1 \mid 32, R)$ super Lie algebra. Each component $M_{Q}{ }^{R}$ of the $33 \times 33$ supermatrix $M$ is promoted to an $N \times N$ matrix and can be expanded in terms of Gell-Mann matrices:

$$
M_{Q}{ }^{R}=\sum_{a=1}^{N^{2}} t^{a}\left(M^{a}\right)_{Q}{ }^{R} .
$$

This action (4.18) is invariant under a tensor product of two gauge groups

$$
M \Rightarrow M+[u, M]
$$

where

$$
u \in g l(1 \mid 32, R) \otimes g l(N, R) \text { or } u(1 \mid 16,16) \otimes u(N) .
$$

Hence the action is invariant under local (or gauged) $u(1 \mid 16,16)$ or $g l(1 \mid 32, R)$ symmetry. That is, the $u(1 \mid 16,16)$ symmetry and $u(N)$ symmetry (or $g l(1 \mid 32, R)$ and $g l(N, R)$ ) are coupled. Not only the bosonic but the fermionic symmetries are also gauged. In this sense this action is considered as a matrix regularization of 11-dimensional supergravity if we can

successfully treat this model. In terms of the components of $M=\left(\begin{array}{cc}m & \psi \\ i \bar{\phi} & v\end{array}\right)$, the action becomes

$$
I=\frac{1}{g^{2}} \operatorname{Tr}_{N \times N}\left(\operatorname{tr}_{32 \times 32}\left(m^{3}\right)-3 i \bar{\phi} m \psi-3 i \bar{\phi} \psi v-v^{3}\right)
$$


In both cases of $u(1 \mid 16,16)$ model and $g l(1 \mid 32, R)$ model, there are $64=32+32$ (real) supercharges. The action is not invariant under the space-time translation which was identified with a constant shift of bosonic fields in the case of IIB or $\operatorname{ssp}(1 \mid 32, R)$ model, and there are no inhomogeneous supersymmetry in this gauged model. To extract space-time translation, we need another interpretation different from the non-gauged model. Here we adopt the Wigner-Inönü contraction of the $S O(10,1)$ symmetry and identify $S O(9,1)$ rotation and space-time translation generators with the $S O(10,1)$ generators. In other words, we zoom in around the north pole of a 10-dimensional sphere on which $S O(10,1)$ rotations are generated by $\Gamma^{A B}$.

First let us consider a $g l(1 \mid 32, R)$-case. To perform the Wigner-Inönü contraction systematically, it is convenient to add another term to the action

$$
I=\frac{1}{3} \operatorname{Tr}_{N \times N} \operatorname{Str}_{33 \times 33}\left(M^{3}\right)-R^{2} \operatorname{Tr}_{N \times N} S t r_{33 \times 33} M
$$

This action has a classical solution

$$
\langle M\rangle=\left(\begin{array}{cc}
R \Gamma^{\sharp} \otimes \mathbf{1}_{N \times N} & 0 \\
0 & R \otimes \mathbf{1}_{N \times N}
\end{array}\right) .
$$

We take a large $R$ limit, which is equivalent to zooming in around the north pole.

Similarly in the case of $u(1 \mid 16,16)$, we need to consider a quintic action $I_{U(1 \mid 16,16)}=$ $\frac{1}{5} \operatorname{Str}\left(M^{5}\right)-R^{4} \operatorname{Str} M$ in order to have the classical solution,

$$
\langle M\rangle=\left(\begin{array}{cc}
R \Gamma^{\sharp} \otimes \mathbf{1}_{N \times N} & 0 \\
0 & i R \otimes \mathbf{1}_{N \times N}
\end{array}\right) .
$$

We focus on the $g l(1 \mid 32, R)$ type in this section, but the following discussions of the WignerInönü contraction are essentially the same as in the $u(1 \mid 16,16)$ case.

We expand the matrix $M$ around the above classical solution $\langle M\rangle$ :

$$
M=\left(\begin{array}{cc}
m & \psi \\
i \bar{\phi} & v
\end{array}\right)=\langle M\rangle+\tilde{M}=\left(\begin{array}{cc}
R \Gamma^{\sharp} & 0 \\
0 & R
\end{array}\right)+\left(\begin{array}{cc}
\tilde{m} & \psi \\
i \bar{\phi} & \tilde{v}
\end{array}\right) .
$$

The action becomes

$$
I=R\left(\operatorname{tr}_{32 \times 32}\left(\tilde{m}^{2} \Gamma^{\sharp}\right)-\tilde{v}^{2}-i \bar{\phi}\left(1+\Gamma^{\sharp}\right) \psi\right)+\frac{1}{3} \operatorname{tr}_{32 \times 32}\left(\tilde{m}^{3}\right)-\frac{\tilde{v}^{3}}{3}-i(\bar{\phi} \tilde{m} \psi+\tilde{v} \bar{\phi} \psi)
$$

up to a constant. In the next subsection, we investigate the model in the large $R$ limit. 


\subsection{Wigner-Inönü contraction and supersymmetry}

In the background proportional to $\Gamma^{\sharp}$, it is convenient to decompose bosonic fields into even and odd rank fields with respect to 10 -dimensional $\gamma$-matrices:

$$
\tilde{m}=m_{e}+m_{o}
$$

where $m_{e}$ is given by

$$
\begin{aligned}
m_{e} & =Z \mathbf{1}+W \Gamma^{\sharp}+\frac{1}{2}\left(C_{\mu_{1} \mu_{2}} \Gamma^{\mu_{1} \mu_{2}}+D_{\mu_{1} \mu_{2}} \Gamma^{\mu_{1} \mu_{2} \sharp}\right) \\
& +\frac{1}{4 !}\left(G_{\mu_{1} \cdots \mu_{4}} \Gamma^{\mu_{1} \cdots \mu_{4}}+H_{\mu_{1} \cdots \mu_{4}} \Gamma^{\mu_{1} \cdots \mu_{4} \sharp}\right)
\end{aligned}
$$

and $m_{o}$ by

$$
\begin{aligned}
m_{o} & =\frac{1}{2}\left(A_{\mu}^{(+)} \Gamma^{\mu}\left(1+\Gamma^{\sharp}\right)+A_{\mu}^{(-)} \Gamma^{\mu}\left(1-\Gamma^{\sharp}\right)\right) \\
& +\frac{1}{2 \times 3 !}\left(E_{\mu_{1} \mu_{2} \mu_{3}}^{(+)} \Gamma^{\mu_{1} \mu_{2} \mu_{3}}\left(1+\Gamma^{\sharp}\right)+E_{\mu_{1} \mu_{2} \mu_{3}}^{(-)} \Gamma^{\mu_{1} \mu_{2} \mu_{3}}\left(1-\Gamma^{\sharp}\right)\right) \\
& +\frac{1}{5 !}\left(I_{\mu_{1} \cdots \mu_{5}}^{(+)} \Gamma^{\mu_{1} \cdots \mu_{5}}\left(1+\Gamma^{\sharp}\right)+I_{\mu_{1} \cdots \mu_{5}}^{(-)} \Gamma^{\mu_{1} \cdots \mu_{5}}\left(1-\Gamma^{\sharp}\right)\right) .
\end{aligned}
$$

We further decompose the $m_{o}$ into $m_{o}^{( \pm)}$according to the $( \pm)$in the above decomposition: $m_{o}=m_{o}^{(+)}+m_{o}^{(-)}$. Fermionic fields are also decomposed according to their chiralities: $\psi_{L, R}=\frac{1 \pm \Gamma^{\sharp}}{2} \psi$. The action then becomes

$$
\begin{aligned}
I & =R\left(\operatorname{tr}_{32 \times 32}\left(m_{e}^{2} \Gamma^{\sharp}\right)-\tilde{v}^{2}-2 i \bar{\phi}_{R} \psi_{L}\right)+\operatorname{tr}_{32 \times 32}\left(\frac{1}{3} m_{e}^{3}+m_{e} m_{o}^{2}\right) \\
& -i\left(\bar{\phi}_{R}\left(m_{e}+\tilde{v}\right) \psi_{L}+\bar{\phi}_{L}\left(m_{e}+\tilde{v}\right) \psi_{R}+\bar{\phi}_{L} m_{o} \psi_{L}+\bar{\phi}_{R} m_{o} \psi_{R}\right)-\frac{1}{3} \tilde{v}^{3} .
\end{aligned}
$$

Since the quadratic term is proportional to $R$, we first rescale $\tilde{v}, m_{e}, \phi_{R}$ and $\psi_{L}$ as $R^{-1 / 2}$. Then, in order to make terms containing other fields such as $t r_{32 \times 32}\left(m_{e} m_{o}^{2}\right)$ finite in the large $R$ limit, we have to rescale the other fields as $R^{1 / 4}$. The rescalings are summarized as

$$
\begin{aligned}
& m=R \Gamma^{\sharp}+\tilde{m}=R \Gamma^{\sharp}+R^{-\frac{1}{2}} m_{e}^{\prime}+R^{\frac{1}{4}} m_{o}^{\prime}, \quad v=R+\tilde{v}=R+R^{-\frac{1}{2}} v^{\prime}, \\
& \psi=\psi_{L}+\psi_{R}=R^{-\frac{1}{2}} \psi_{L}^{\prime}+R^{\frac{1}{4}} \psi_{R}^{\prime}, \quad \bar{\phi}=\bar{\phi}_{L}+\bar{\phi}_{R}=R^{\frac{1}{4}} \bar{\phi}_{L}^{\prime}+R^{-\frac{1}{2}} \bar{\phi}_{R}^{\prime} .
\end{aligned}
$$

In terms of these rescaled fields, we can rewrite the action, by dropping terms with a negative power of $R$, as

$$
\begin{aligned}
I= & \left(t_{32 \times 32}\left(m_{e}^{\prime 2} \Gamma^{\sharp}\right)-v^{\prime 2}+\operatorname{tr}_{32 \times 32}\left(m_{e}^{\prime} m_{o}^{\prime 2}\right)\right) \\
& +i\left(-2 \bar{\phi}_{R}^{\prime} \psi_{L}^{\prime}+\bar{\phi}_{L}^{\prime}\left(m_{e}^{\prime}+v^{\prime}\right) \psi_{R}^{\prime}+\bar{\psi}_{L}^{\prime} m_{o}^{\prime} \psi_{L}^{\prime}+\bar{\phi}_{R}^{\prime} m_{o}^{\prime} \psi_{R}^{\prime}\right) .
\end{aligned}
$$


Since only the fields $v^{\prime}, m_{e}^{\prime}, \phi_{R}^{\prime}$ and $\psi_{L}^{\prime}$ have quadratic terms, we may integrate them and obtain an effective action for the other fields. Before performing the integration, let us first look at the supersymmetry structure in order to see how we can obtain space-time supersymmetry in our model. We can also see the above scalings are consistent with supersymmetries.

The 10-dimensional space-time translation around the north pole is generated by $\Gamma_{\mu \sharp}$. Since $R$ is interpreted as the radius of $S^{10}$, space-time translation generator should be identified with $P_{\mu}=\frac{1}{R} \Gamma_{\mu \sharp}$. On the other hand, a commutator of two supercharges $Q_{\chi \epsilon}=\left(\begin{array}{cc}0 & \chi \\ i \bar{\epsilon} & 0\end{array}\right)$ and $Q_{\rho \eta}=\left(\begin{array}{cc}0 & \rho \\ i \bar{\eta} & 0\end{array}\right)$ becomes

$$
\left[Q_{\chi \epsilon}, Q_{\rho \eta}\right]=\left(\begin{array}{cc}
i(\chi \bar{\eta}-\rho \bar{\epsilon}) & 0 \\
0 & i(\bar{\epsilon} \rho-\bar{\eta} \chi)
\end{array}\right),
$$

which contains the translation $P_{\mu}$ besides other $g l(32, R)$ bosonic generators. In this way, the homogeneous supersymmetry in gauged models is considered as 10-dimensional space-time supersymmetry.

In addition to scaling the fields as above, we need to scale gauge parameters of $g l(1 \mid 32, R)$. Writing the gauge parameter $h$ by

$$
h=\left(\begin{array}{cc}
a & \chi \\
i \bar{\epsilon} & b
\end{array}\right)
$$

the field $M$ is transformed as

$$
\begin{aligned}
\delta \tilde{M} & =[h, M]=[h,\langle M\rangle+\tilde{M}] \\
& =\left(\begin{array}{cc}
{\left[a, \tilde{m}+R \Gamma^{\sharp}\right]+i(\chi \bar{\phi}-\psi \bar{\epsilon})} & -\left(\tilde{m}+R \Gamma^{\sharp}\right) \chi+a \psi-b \psi+\chi \tilde{v} \\
i \bar{\epsilon}\left(\tilde{m}+R \Gamma^{\sharp}\right)-i(\bar{\phi} a+\tilde{v} \bar{\epsilon})+i b \bar{\phi} & i(\bar{\epsilon} \psi-\bar{\phi} \chi)+[b, \tilde{v}]
\end{array}\right),
\end{aligned}
$$

where inhomogeneous terms come from $[h,\langle M\rangle]$. The inhomogeneous term for $m_{o}$ should survive after taking the large $R$ limit, since space-time translations for $A_{\mu}^{( \pm)}$are included there. Decomposing the bosonic gauge parameter $a$ into $a_{o}$ and $a_{e}$ similarly to (4.28), the inhomogeneous part of $\delta m_{o}$ is given by $\delta m_{o}=\left[a_{o}, R \Gamma^{\sharp}\right]$. Since we have rescaled $m_{o}=R^{1 / 4} m_{o}^{\prime}$, we should rescale $a_{o}$ as $R^{-3 / 4}$ so as to make this inhomogeneous term finite in the large $R$ limit. On the other hand, $S O(9,1)$ rotation generated by $\Gamma_{\mu \nu}$ is included in $a_{e}$ and it transforms even (odd) rank fields into themselves. The gauge parameter $a_{e}$ is, therefore, not necessary to be rescaled. Similar arguments can be applied to supersymmetries and we finally obtain the following rescalings

$$
h=\left(\begin{array}{cc}
a_{e}^{\prime}+R^{-\frac{3}{4}} a_{o}^{\prime} & \chi_{L}^{\prime}+R^{-\frac{3}{4}} \chi_{R}^{\prime} \\
i\left(R^{-\frac{3}{4}} \bar{\epsilon}_{L}^{\prime}+\bar{\epsilon}_{R}^{\prime}\right) & b^{\prime}
\end{array}\right) .
$$


Under this gauge transformation, each field transforms in the large $R$ limit as

$$
\begin{aligned}
\delta m_{o}^{\prime} & =\underline{\left[a_{o}, \Gamma^{\sharp}\right]}+\left[a_{e}^{\prime}, m_{o}^{\prime}\right]+i\left(\chi_{L}^{\prime} \bar{\phi}_{L}^{\prime}-\psi_{R}^{\prime} \bar{\epsilon}_{R}^{\prime}\right), \\
\delta \psi_{R}^{\prime} & =\underline{2 \chi_{R}^{\prime}}+\left(a_{e}^{\prime} \psi_{R}^{\prime}-b \psi_{R}^{\prime}-\left(m_{o}^{\prime} \chi_{L}^{\prime}\right)\right), \\
\delta \bar{\phi}_{L}^{\prime} & =\underline{-2 \bar{\epsilon}_{L}^{\prime}}+\left(\left(\bar{\epsilon}_{R}^{\prime} m_{o}^{\prime}\right)+b^{\prime} \bar{\phi}_{L}^{\prime}-\bar{\phi}_{L}^{\prime} a_{e}^{\prime}\right), \\
\delta m_{e}^{\prime} & =\left[a_{e}, m_{e}^{\prime}\right]+\left[a_{o}, m_{o}^{\prime}\right]+i\left(\chi_{L}^{\prime} \bar{\epsilon}_{R}^{\prime}+\chi_{R}^{\prime} \bar{\epsilon}_{L}^{\prime}\right)-i\left(\psi_{L}^{\prime} \bar{\epsilon}_{R}^{\prime}+\chi_{R}^{\prime} \bar{\epsilon}_{L}^{\prime}\right), \\
\delta \psi_{L}^{\prime} & =-\left(m_{o}^{\prime} \chi_{R}^{\prime}+m_{e}^{\prime} \chi_{L}\right)+\left(a_{o} \psi_{R}^{\prime}+a_{e} \psi_{L}^{\prime}\right)-b^{\prime} \psi_{L}^{\prime}+v^{\prime} \chi_{L}^{\prime}, \\
\delta \bar{\phi}_{R}^{\prime} & =\left(\bar{\epsilon}_{R}^{\prime} m_{e}^{\prime}+\bar{\epsilon}_{L}^{\prime} m_{o}^{\prime}\right)+b^{\prime} \bar{\phi}_{R}^{\prime}-\left(\bar{\phi}_{L}^{\prime} a_{o}^{\prime}+\bar{\phi}_{R}^{\prime} a_{e}^{\prime}\right)-v^{\prime} \bar{\epsilon}_{R}^{\prime}, \\
\delta v^{\prime} & =i\left(\bar{\epsilon}_{R}^{\prime} \psi_{L}^{\prime}+\bar{\epsilon}_{L}^{\prime} m_{o}^{\prime}\right)-i\left(\bar{\phi}_{R}^{\prime} \chi_{L}^{\prime}+\bar{\phi}_{L}^{\prime} \chi_{R}^{\prime}\right)+\left[b^{\prime}, v^{\prime}\right] .
\end{aligned}
$$

The underlined terms are inhomogeneous transformations. The other transformations are homogeneous and linear in fields. As we have seen in the action, the fields that do not receive inhomogeneous transformations, that is, $m_{e}^{\prime}, v^{\prime}, \psi_{L}^{\prime}$ and $\phi_{R}^{\prime}$ contain quadratic terms and can be integrated out by Gaussian integration. An important point is that the transformations of the first three fields $m_{o}^{\prime}, \psi_{R}^{\prime}$ and $\phi_{L}^{\prime}$ do not include the other fields in the right hand side. This means that these transformation rules are not changed after integrating out the other fields, $m_{e}^{\prime}, \psi_{L}^{\prime}, \phi_{R}^{\prime}$ and $v^{\prime}$.

Now let us obtain the effective action by integrating out $v^{\prime}, m_{e}^{\prime}, \psi_{L}^{\prime}$ and $\phi_{R}^{\prime}$. The integration can be easily done and the effective action vanishes!

$$
W=-\frac{1}{4} \operatorname{tr}_{32 \times 32}\left(\Gamma^{\sharp}\left\{m_{o}^{\prime 2}+i\left(\psi_{R}^{\prime} \bar{\phi}_{L}^{\prime}\right)\right\}^{2}\right)-\frac{1}{4}\left(\bar{\phi}_{L}^{\prime} \psi_{R}\right)^{2}+\frac{i}{2}\left(\bar{\phi}_{L}^{\prime} m_{o}^{\prime 2} \psi_{R}^{\prime}\right)=0 .
$$

Here we have used

$$
\operatorname{tr}_{32 \times 32}\left(\Gamma^{\sharp} m_{o}^{\prime 4}\right)=0 .
$$

The reason for the vanishment of the effective action can be understood from the symmetry point of view. Since the transformations for $m_{o}^{\prime}, \psi_{R}^{\prime}$ and $\phi_{L}^{\prime}$ do not include the other integrated fields, they are not changed after integration. Therefore the effective action must be invariant under the same transformations, which include $U(N)$ dependent shifts of them, not restricted to constant shifts. The only action invariant under such transformations is a trivial one. In this sense, this model is a topological model of fields $m_{o}^{\prime}, \psi_{R}^{\prime}$ and $\phi_{L}^{\prime}$.

Although the action vanishes, we go on to investigate the supersymmetry structures. If we decompose the boson fields $m_{o}^{\prime}$ into $m_{o}^{\prime( \pm)}$, we obtain the following transformations

$$
\begin{aligned}
& \delta m_{o}^{\prime(+)}=-i\left(\psi_{R}^{\prime} \bar{\epsilon}_{R}^{\prime}\right), \quad \delta m_{o}^{\prime(-)}=i\left(\chi_{L}^{\prime} \bar{\phi}_{L}^{\prime}\right), \\
& \delta \psi_{R}^{\prime}=2 \chi_{R}^{\prime}-\left(m_{o}^{\prime(+)} \chi_{L}^{\prime}\right), \quad \delta \bar{\phi}_{L}^{\prime}=-2 \bar{\epsilon}_{L}^{\prime}+\left(\bar{\epsilon}_{R}^{\prime} m_{o}^{\prime(-)}\right),
\end{aligned}
$$


and we can see that left and right handed fields are decoupled. We have two pairs of fields, $\left(m_{o}^{(+)}\right.$and $\left.\psi_{R}\right)$ and $\left(m_{o}^{(-)}\right.$and $\left.\phi_{L}\right)$. This pairing is the same as that of $\operatorname{osp}(1 \mid 32, R)$ model. In this case, since the effective action vanishes we do not have the problem of compatibility with the pairing in the action. More explicitly in terms of $A_{\mu}{ }^{( \pm)}$fields, the homogeneous supersymmetry transformations become

$$
\delta_{\chi_{L}}^{(1)} A_{i}^{(-)}=-\frac{i}{16} \bar{\phi}_{L}^{\prime} \Gamma_{i} \chi_{L}, \quad \delta_{\epsilon_{R}}^{(1)} A_{i}^{\prime(+)}=\frac{i}{16} \bar{\epsilon}_{R} \Gamma_{i} \psi_{R}^{\prime}
$$

which are the same as those of IIB matrix model. On the other hand, transformations for fermions are different from those of IIB matrix model. Instead of the commutators $\left[A_{\mu}^{( \pm)}, A_{\nu}^{( \pm)}\right]$, they are proportional to a single $A_{\mu}^{( \pm)}$and accordingly supersymmetry parameters with opposite chirality. Because of this reason, it seems difficult to interpret IIB matrix model as a gauge fixed version of the gauged matrix model investigated here. But such gauged models are interesting from various points of view, especially the existence of local Lorentz invariance, and it is worth further investigations. More analysis will be reported elsewhere.

\section{Conclusions and discussions}

In this paper, we have investigated several matrix models based on super Lie algebra. First we have studied $\operatorname{sep}(1 \mid 32, R)$ matrix model. This model is considered as an 11-dimensional model and contains twice as many fermionic degrees of freedom as IIB matrix model. The model is invariant under global $\operatorname{osp}(1 \mid 32, R)$ symmetry and $U(N)$ gauge symmetry. It is also invariant under a constant shift of fields. Combining the $\operatorname{osp}(1 \mid 32, R)$ and the constant shifts, we obtain space-time algebras including space-time supersymmetries. In this sense, this model is a natural generalization of IIB matrix model. Since this model has twice as many fermions, we need to integrate half of the degrees of freedom. We have given an identification of the fields in this model with the fields in IIB matrix model from the view point of the supersymmetry structures. We have also discussed a possibility to induce IIB matrix model by integrating out the unnecessary fields.

In the latter half of this paper, we have studied the gauged matrix models with local Lorentz symmetry. First we have shown that the unitary transformations in noncommutative gauge theories contain much larger symmetries than the ordinary gauge transformations. Especially, local coordinate transformations can be described within this gauge transformations. This is understandable from the D-brane point of view. If a noncommutative gauge 
theory is considered as an effective low-energy action for D-branes, the action should be invariant under coordinate transformations on the brane. Under this transformation, all the fields $\psi$ and $A_{\mu}$ in gauge theory transform as scalars. More interestingly we have shown that if we expand those fields, not only in terms of $\exp \left(i \hat{p}_{\mu} k_{\mu}\right)$ but as a power series of $\hat{p}_{\mu}$, we can obtain higher rank fields which transform as tensors under this coordinate transformations. However, the original $S O(9,1)$ indices are completely decoupled from the internal diffeomorphism.

In the final section, we have considered a model with local $S O(9,1)$ symmetry by extending the $\operatorname{osp}(1 \mid 32, R)$ algebra to $u(1 \mid 16,16)$ or $g l(1 \mid 32, R)$ super Lie algebras. We have enhanced the global osp $(1 \mid 32, R)$ to local symmetries, but lost the invariance under constant shifts of fields and we need a different interpretation of space-time translation. We have adopted the Wigner-Inönü contraction and extracted 10-dimensional space-time translation from $S O(10,1)$ rotations. We have then identified how to scale the fields in order to obtain the correct 10-dimensional theory in the large radius limit. Since this model contains four times as many fermionic fields as IIB matrix model, we need to integrate out half of them first and then restrict the fermions further to be halved. But after integrating the first half of the fields, the effective action was shown to vanish. This is because the resultant action should be invariant under an arbitrary shift of the fields, not restricted to a constant shift. We can interpret the final model as a topological model of IIB matrix model. This type of the topological model was studied in [20]. It is also interesting to investigate such a possibility from the gauged matrix model point of view.

\section{A Supermatrices}

Here we briefly summarize our conventions of super Lie algebra. First the complex conjugate of Grassmann variables is defined by

$$
(\alpha \beta)^{*}=\beta^{*} \alpha^{*}
$$

For a supermatrix $M=\left(\begin{array}{ll}a & \beta \\ \gamma & d\end{array}\right)$ with bosonic matrices $a, d$ and fermionic matrices $\beta, \gamma$, we define its transpose, hermitian conjugate and complex conjugate as follows:

$$
{ }^{T} M=\left(\begin{array}{cc}
{ }^{T} a & -^{T} \gamma \\
{ }^{T} \beta & { }^{T} d
\end{array}\right), \quad M^{\dagger}=\left(\begin{array}{cc}
a^{\dagger} & \gamma^{\dagger} \\
\beta^{\dagger} & d^{\dagger}
\end{array}\right), \quad M^{*}=\left({ }^{T} M\right)^{\dagger}=\left(\begin{array}{cc}
a^{*} & \beta^{*} \\
-\gamma^{*} & d^{*}
\end{array}\right) .
$$

From these definitions, we have the following relations

$$
{ }^{T} M=\left(M^{*}\right)^{\dagger} \neq\left(M^{\dagger}\right)^{*}, \quad M^{\dagger}={ }^{T}\left(M^{*}\right) \neq\left({ }^{T} M\right)^{*} .
$$


Note also that ${ }^{T}\left({ }^{T} M\right) \neq M$ while $\left(M^{\dagger}\right)^{\dagger}=M$ and $\left(M^{*}\right)^{*}=M$. For two supermatrices $M_{1}$ and $M_{2}$, we have the identities ${ }^{T}\left(M_{1} M_{2}\right)={ }^{T} M_{2}{ }^{T} M_{1},\left(M_{1} M_{2}\right)^{\dagger}=M_{2}^{\dagger} M_{1}^{\dagger}$ and $\left(M_{1} M_{2}\right)^{*}=M_{1}^{*} M_{2}^{*}$.

\section{B Proof against a perturbative generation for $W$ and $A_{\mu}^{(-)}$propagators}

In this appendix we prove that it is impossible to generate propagators for $W$ and $A_{\mu}^{(-)}$ fields through perturbative calculations in $\operatorname{osp}(1 \mid 32, R)$ matrix model discussed in section 2. Of course, this proof does not exclude nonperturbative appearance of propagators for them. First we assign charges $(1,0,-1)$ to the bosonic fields $\left(m_{e}, m_{o}^{(+)}, m_{o}^{(-)}\right)$and $(0,1 / 2)$ to $\left(\psi_{L}, \psi_{R}\right)$. As we can see from the action (2.30), every three point vertex has charge $3,3 / 2$ or 0 . Similarly in the background of (2.46), propagators appear at tree level for $\left\langle m_{e} m_{o}^{(-)}\right\rangle$and $\left\langle\psi_{L} \bar{\psi}_{L}\right\rangle$ and they have charges 1 and 0 respectively. On the other hand, two point function $\langle W W\rangle$ which is included in $\left\langle m_{e} m_{e}\right\rangle$ or $\left\langle A_{\mu}^{(-)} A_{\nu}^{(-)}\right\rangle$in $\left\langle m_{o}^{(-)} m_{o}^{(-)}\right\rangle$has charge 2 or -2 respectively. Hence it is clearly impossible to generate these two point functions perturbatively no matter how we combine the above vertices and tree level propagators. 


\section{References}

[1] N. Ishibashi, H. Kawai, Y. Kitazawa and A. Tsuchiya, Nucl. Phys. B 498, 467 (1997) hep-th/9612115.

[2] M. Fukuma, H. Kawai, Y. Kitazawa and A. Tsuchiya, Nucl. Phys. B 510, 158 (1998) hep-th/9705128.

[3] T. Eguchi and H. Kawai, Phys. Rev. Lett. 48, 1063 (1982). G. Parisi, Phys. Lett. B 112, 463 (1982). D. J. Gross and Y. Kitazawa, Nucl. Phys. B 206, 440 (1982). G. Bhanot, U. M. Heller and H. Neuberger, Phys. Lett. B 113, 47 (1982).

[4] H. Aoki, S. Iso, H. Kawai, Y. Kitazawa and T. Tada, Prog. Theor. Phys. 99, 713 (1998) hep-th/9802085.

[5] T. Hotta, J. Nishimura and A. Tsuchiya, Nucl. Phys. B 545, 543 (1999) [hepth/9811220]. J. Ambjorn, K. N. Anagnostopoulos, W. Bietenholz, T. Hotta and J. Nishimura, JHEP0007, 013 (2000) hep-th/0003208, JHEP0007, 011 (2000) [hepth/0005147, hep-th/0101084.

[6] H. Aoki, S. Iso, H. Kawai, Y. Kitazawa, A. Tsuchiya and T. Tada, Prog. Theor. Phys. Suppl. 134, 47 (1999) hep-th/9908038.

[7] M. Li, Nucl. Phys. B 499, 149 (1997) hep-th/9612222],

H. Aoki, N. Ishibashi, S. Iso, H. Kawai, Y. Kitazawa and T. Tada, Nucl. Phys. B 565, 176 (2000) hep-th/9908141, N. Ishibashi, S. Iso, H. Kawai and Y. Kitazawa, Nucl. Phys. B 573, 573 (2000) hep-th/9910004.

[8] S. Iso, H. Kawai and Y. Kitazawa, Nucl. Phys. B 576, 375 (2000) hep-th/0001027.

[9] S. Iso, Y. Kimura, K. Tanaka and K. Wakatsuki, hep-th/0101102.

[10] S. Iso and H. Kawai, Int. J. Mod. Phys. A 15, 651 (2000) hep-th/9903217.

[11] E. Cremmer, B. Julia and J. Scherk, Phys. Lett. B76, 409 (1978).

[12] J. W. van Holten and A. Van Proeyen, J. Phys. AA15, 3763 (1982).

[13] P. K. Townsend, hep-th/9712004. 
[14] E. Bergshoeff and A. Van Proeyen, hep-th/0010194.

[15] L. Smolin, Nucl. Phys. B591, 227 (2000) hep-th/0002009. L. Smolin, hep-th/0006137.

[16] J. Ambjorn, Y. M. Makeenko, J. Nishimura and R. J. Szabo, JHEP9911, 029 (1999) hep-th/9911041, JHEP0005, 023 (2000) hep-th/0004147,

[17] G. Parisi, Phys. Lett. 112B (1982) 463.

[18] S. R. Das and S. Rey, Nucl. Phys. B590, 453 (2000) hep-th/0008042].

[19] D. J. Gross, A. Hashimoto and N. Itzhaki, hep-th/0008075.

[20] S. Hirano and M. Kato, Prog. Theor. Phys. 98, 1371 (1997) hep-th/9708039. 